\title{
Quasiequilibrium black hole-neutron star binaries in general relativity
}

\author{
Keisuke Taniguchi, ${ }^{1}$ Thomas W. Baumgarte,, , Joshua A. Faber, ${ }^{1}$ and Stuart L. Shapiro ${ }^{1, \text { 由 }}$ \\ ${ }^{1}$ Department of Physics, University of Illinois at Urbana-Champaign, Urbana, Illinois 61801, USA \\ ${ }^{2}$ Department of Physics and Astronomy, Bowdoin College, Brunswick, Maine 04011, USA
}

(Dated: October 24, 2007)

\begin{abstract}
We construct quasiequilibrium sequences of black hole-neutron star binaries in general relativity. We solve Einstein's constraint equations in the conformal thin-sandwich formalism, subject to black hole boundary conditions imposed on the surface of an excised sphere, together with the relativistic equations of hydrostatic equilibrium. In contrast to our previous calculations we adopt a flat spatial background geometry and do not assume extreme mass ratios. We adopt a $\Gamma=2$ polytropic equation of state and focus on irrotational neutron star configurations as well as approximately nonspinning black holes. We present numerical results for ratios of the black hole's irreducible mass to the neutron star's ADM mass in isolation of $M_{\mathrm{irr}}^{\mathrm{BH}} / M_{\mathrm{ADM}, 0}^{\mathrm{NS}}=1,2,3,5$, and 10 . We consider neutron stars of baryon rest mass $M_{\mathrm{B}}^{\mathrm{NS}} / M_{\mathrm{B}}^{\max }=83 \%$ and $56 \%$, where $M_{\mathrm{B}}^{\max }$ is the maximum allowed rest mass of a spherical star in isolation for our equation of state. For these sequences, we locate the onset of tidal disruption and, in cases with sufficiently large mass ratios and neutron star compactions, the innermost stable circular orbit. We compare with previous results for black hole-neutron star binaries and find excellent agreement with third-order post-Newtonian results, especially for large binary separations. We also use our results to estimate the energy spectrum of the outgoing gravitational radiation emitted during the inspiral phase for these binaries.
\end{abstract}

PACS numbers: 04.30.Db, 04.25.Dm, 04.40.Dg

\section{INTRODUCTION}

Coalescing compact binaries composed of neutron stars and/or black holes are among the most promising sources of gravitational waves for ground-based laser interferometers [1, 2, 3, 4]. Observations of short gamma-ray bursts (SGRBs) by the Swift and HETE-2 satellites (see, e.g. 5] and references therein) suggest that their central engine may well be the merger remnant of compact binaries that contain a neutron star, namely the remnants of black hole-neutron star (BHNS) binaries or binary neutron star (BNS) systems [6, 7, 8, 9]. Motivated by these considerations, significant theoretical effort has gone into the modeling of these binaries.

So far, most studies of BHNS binaries have been performed within the framework of Newtonian gravity in either some or all aspects of the calculation (see, e.g. 10 , $11,12,13,14,15,16,17,18$ for quasiequilibrium calculations and 19, 20, 21, 22, 23, 24, 25] for dynamical simulations). More recently, several groups have initiated studies of BHNS binaries in a fully relativistic framework, both for quasiequilibrium models [26, 27, 28, 29, 30] and dynamical simulations [7, 31, 32, 33, 34].

In our own effort, we initially studied BHNS quasiequilibrium models under the assumption of extreme mass ratios, where the mass of the black hole is much greater than that of the neutron star [7, 27, 28, 31]. This assumption is very appealing computationally and therefore a

\footnotetext{
*Also at: Department of Physics, University of Illinois at UrbanaChampaign, Urbana, Illinois 61801, USA

${ }^{\dagger}$ Also at: Department of Astronomy and NCSA, University of Illinois at Urbana-Champaign, Urbana, Illinois 61801, USA
}

natural first step in a systematic study of these binaries, but breaks down for the astrophysically more interesting comparable-mass binaries. For the latter, the neutron star is tidally disrupted outside of the black hole's innermost stable circular orbit (ISCO) (see, e.g. [18]) and may form an accretion disk, which is considered crucial for launching of a gamma-ray burst. The observation of such a process would provide a wealth of astrophysical information [35]. In a subsequent study of quasiequilibrium BHNS models 29], we therefore relaxed the assumption of extreme mass ratios and studied comparable-mass binaries (see also [30, 34]).

We construct quasiequilibrium models by solving Einstein's constraint equations in the conformal thinsandwich formalism (see, e.g. [36, 37, 38]). These equations constrain only some of the gravitational fields; others, namely the conformal background solution, are freely specifiable and have to be chosen before the equations can be solved (see Section $\amalg \mathrm{A}$ below). In our previous studies 27, 28, 29] we chose a background solution describing a single black hole in Kerr-Schild coordinates (in [28] we also include results for a spatially flat background for extreme mass ratio binaries). This seemed appealing in a number of ways; for example, it allows for black hole spin and the coordinate system extends smoothly into the black hole interior. However, in 29 we found sizable deviations from post-Newtonian (PN) results which we could attribute, at least in part, to the choice of the background solution [28, 30]. Moreover, the recent black hole boundary conditions of Cook and Pfeiffer [39] (see also [40]) allow for black hole rotation and penetrating coordinates even for a spatially flat background solution, which simplifies the equations significantly.

In this paper we therefore revisit quasiequilibrium 
models of BHNS binaries. We now use a spatially flat background solution instead of a Kerr-Schild metric (compare [30]), and we also adopt a new decomposition of the field variables which significantly reduces the numerical error that was still present in [29] (see Appendix Ad). We construct sequences of BHNS binaries in quasicircular orbits for fixed black hole irreducible masses and neutron star baryon rest masses, and focus on irrotational neutron stars orbiting approximately nonspinning black holes (we will implement a more accurate condition for nonspinning black holes in the future, following [40]). We find excellent agreement with post-Newtonian results for large binary separations. We track cusp formation to locate the onset of tidal disruption, and, for large mass ratios and neutron star compactions, find turningpoints on a binding energy curve to locate the ISCO. For those sequences that encounter an ISCO we find reasonable agreement with the ISCO of test particles around Schwarzschild black holes.

The paper is organized as follows. We briefly review the basic equations in Section II. We present numerical results in Section III, and provide a comparison with the results of [28, 29, 30] in Section IV. In Section V we briefly summarize our findings.

Throughout this paper we adopt geometric units with $G=c=1$, where $G$ denotes the gravitational constant and $c$ the speed of light. Latin and Greek indices denote purely spatial and spacetime components, respectively.

\section{FORMULATION}

In this Section we briefly review the equations we solve to obtain a BHNS binary in quasiequilibrium. For a more detailed discussion we refer to the review articles [37, 38] as well as Sec. II of [41] for the hydrostatics.

\section{A. Gravitational field equations}

The line element in $3+1$ form is written as

$$
\begin{aligned}
d s^{2} & =g_{\mu \nu} d x^{\mu} d x^{\nu} \\
& =-\alpha^{2} d t^{2}+\gamma_{i j}\left(d x^{i}+\beta^{i} d t\right)\left(d x^{j}+\beta^{j} d t\right),
\end{aligned}
$$

where $g_{\mu \nu}$ is the spacetime metric, $\alpha$ the lapse function, $\beta^{i}$ the shift vector, and $\gamma_{i j}$ the spatial metric induced on a spatial slice $\Sigma$. The spatial metric $\gamma_{i j}$ is further decomposed according to $\gamma_{i j}=\psi^{4} \tilde{\gamma}_{i j}$, where $\psi$ denotes the conformal factor and $\tilde{\gamma}_{i j}$ the background spatial metric. We also decompose the extrinsic curvature $K^{i j}$ into a trace $K$ and a traceless part $\tilde{A}^{i j}$ according to

$$
K^{i j}=\psi^{-10} \tilde{A}^{i j}+\frac{1}{3} \gamma^{i j} K .
$$

The Hamiltonian constraint then becomes

$$
\tilde{\nabla}^{2} \psi=-2 \pi \psi^{5} \rho+\frac{1}{8} \psi \tilde{R}+\frac{1}{12} \psi^{5} K^{2}-\frac{1}{8} \psi^{-7} \tilde{A}_{i j} \tilde{A}^{i j} .
$$

Here $\tilde{\nabla}^{2}=\tilde{\gamma}^{i j} \tilde{\nabla}_{i} \tilde{\nabla}_{j}$ is the covariant Laplace operator, $\tilde{\nabla}_{i}$ the covariant derivative, $\tilde{R}_{i j}$ the Ricci tensor, and $\tilde{R}=\tilde{\gamma}^{i j} \tilde{R}_{i j}$ the scalar curvature, all associated with the conformal background metric $\tilde{\gamma}_{i j}$.

We employ the conformal thin-sandwich decomposition of the Einstein equations 36]. In this decomposition, we use the evolution equation for the spatial metric to express the traceless part of the extrinsic curvature in terms of the time derivative of the background metric, $\tilde{u}_{i j} \equiv \partial_{t} \tilde{\gamma}_{i j}$, and the gradients of the shift vector. Under the assumption of equilibrium, i.e., $\tilde{u}_{i j}=0$ in a corotating coordinate system, the traceless part of the extrinsic curvature reduces to

$$
\tilde{A}^{i j}=\frac{\psi^{6}}{2 \alpha}\left(\tilde{\nabla}^{i} \beta^{j}+\tilde{\nabla}^{j} \beta^{i}-\frac{2}{3} \tilde{\gamma}^{i j} \tilde{\nabla}_{k} \beta^{k}\right) .
$$

Inserting Eq. (4) into the momentum constraint we obtain

$$
\begin{aligned}
& \tilde{\nabla}^{2} \beta^{i}+\frac{1}{3} \tilde{\nabla}^{i}\left(\tilde{\nabla}_{j} \beta^{j}\right)+\tilde{R}_{j}^{i} \beta^{j} \\
& =16 \pi \alpha \psi^{4} j^{i}+2 \tilde{A}^{i j} \tilde{\nabla}_{j}\left(\alpha \psi^{-6}\right)+\frac{4}{3} \alpha \tilde{\gamma}^{i j} \tilde{\nabla}_{j} K .
\end{aligned}
$$

For the construction of quasiequilibrium data it is also reasonable to assume $\partial_{t} K=0$ in a corotating coordinate system. The trace of the evolution equation for the extrinsic curvature then yields

$$
\begin{aligned}
\tilde{\nabla}^{2} \alpha= & 4 \pi \alpha \psi^{4}(\rho+S)+\frac{1}{3} \alpha \psi^{4} K^{2}+\psi^{4} \beta^{i} \tilde{\nabla}_{i} K \\
& +\alpha \psi^{-8} \tilde{A}_{i j} \tilde{A}^{i j}-2 \tilde{\gamma}^{i j} \tilde{\nabla}_{i} \alpha \tilde{\nabla}_{j} \ln \psi .
\end{aligned}
$$

Equations (3), (5) and (6) provide equations for the lapse function $\alpha$, the shift vector $\beta^{i}$, and the conformal factor $\psi$, while $\tilde{A}^{i j}$ can be found from Eq. (44). The conformally related spatial metric $\tilde{\gamma}_{i j}$ and the trace of the extrinsic curvature $K$ remain freely specifiable, and have to be chosen before we can solve the above equations (note that we have already set to zero the time derivatives of these quantities, which are also freely specifiable). In [29] we identified these quantities with the corresponding quantities for a Schwarzschild black hole in Kerr-Schild coordinates. Instead, we now assume a flat background $\tilde{\gamma}_{i j}=\eta_{i j}$, where $\eta_{i j}$ denotes a flat spatial metric, and maximal slicing $K=0$. In Cartesian coordinates, Eqs. (3), (5) and (6) then reduce to

$$
\begin{aligned}
& \underline{\Delta} \psi=-2 \pi \psi^{5} \rho-\frac{1}{8} \psi^{-7} \tilde{A}_{i j} \tilde{A}^{i j} \\
& \underline{\Delta} \beta^{i}+\frac{1}{3} \partial^{i}\left(\partial_{j} \beta^{j}\right)=16 \pi \alpha \psi^{4} j^{i}+2 \tilde{A}^{i j} \partial_{j}\left(\alpha \psi^{-6}\right), \\
& \underline{\Delta} \alpha=4 \pi \alpha \psi^{4}(\rho+S)+\alpha \psi^{-8} \tilde{A}_{i j} \tilde{A}^{i j} \\
& \quad-2 \eta^{i j} \partial_{i} \alpha \partial_{j} \ln \psi,
\end{aligned}
$$

where $\underline{\Delta}$ and $\partial_{i}$ denote the flat Laplace operator and the flat partial derivative, while Eq. (4) becomes

$$
\tilde{A}^{i j}=\frac{\psi^{6}}{2 \alpha}\left(\partial^{i} \beta^{j}+\partial^{j} \beta^{i}-\frac{2}{3} \eta^{i j} \partial_{k} \beta^{k}\right) .
$$


For numerical purposes we further decompose the variables and their equations into parts associated with the black hole and the neutron star. For details of this decomposition we refer to Appendix A, but we note that we have found this decomposition crucial for improving the accuracy of the calculations.

The matter terms on the right-hand side of Eqs. (7), (8), and (9) are derived from the projections of the stressenergy tensor $T_{\mu \nu}$ into the spatial slice $\Sigma$. Assuming an ideal fluid, we have

$$
T_{\mu \nu}=\left(\rho_{0}+\rho_{i}+P\right) u_{\mu} u_{\nu}+P g_{\mu \nu},
$$

where $u_{\mu}$ is the fluid 4-velocity, $\rho_{0}$ the baryon rest-mass density, $\rho_{i}$ the internal energy density, and $P$ the pressure. Denoting the future-oriented unit normal to $\Sigma$ as $n_{\mu}$, the relevant projections of $T_{\mu \nu}$ are

$$
\begin{aligned}
& \rho=n_{\mu} n_{\nu} T^{\mu \nu}, \\
& j^{i}=-\gamma_{\mu}^{i} n_{\nu} T^{\mu \nu}, \\
& S_{i j}=\gamma_{i \mu} \gamma_{j \nu} T^{\mu \nu}, \\
& S=\gamma^{i j} S_{i j} .
\end{aligned}
$$

\section{B. Hydrostatic equations}

The matter in the neutron star interior has to satisfy the relativistic equations of hydrodynamics. For stationary configurations, the relativistic Euler equation can be integrated once to yield

$$
h \alpha \frac{\gamma}{\gamma_{0}}=\text { constant }
$$

where $h=\left(\rho_{0}+\rho_{i}+P\right) / \rho_{0}$ is the fluid specific enthalpy, and $\gamma$ and $\gamma_{0}$ are Lorenz factors between the fluid and the rotating frame, and the rotating frame and the inertial frame (see Sec. II.C. of [28] for the definitions). For irrotational fluids, the fluid 3-velocity with respect to the inertial observer, $U^{i}$, can be expressed in terms of the gradient of a velocity potential $\Psi$ as

$$
U^{i}=\frac{\psi^{-4}}{\alpha u^{t} h} \tilde{\nabla}^{i} \Psi,
$$

where $u^{t}$ is the time component of the fluid 4-velocity $u^{\mu}$. Having taken into account the expression of the 3velocity, the equation of continuity becomes

$$
\frac{\rho_{0}}{h} \nabla^{\mu} \nabla_{\mu} \Psi+\left(\nabla^{\mu} \Psi\right) \nabla_{\mu}\left(\frac{\rho_{0}}{h}\right)=0,
$$

where $\nabla_{\mu}$ denotes the covariant derivative associated with $g_{\mu \nu}$. We refer to [41] for a more detailed derivation of the hydrostatic equations, and to [42, 43, 44] for BNS applications.

\section{Equation of state}

We adopt a polytropic equation of state in the form

$$
P=\kappa \rho_{0}^{\Gamma}
$$

where $\Gamma$ denotes the adiabatic index and $\kappa$ a constant. In this paper, we focus on the case $\Gamma=2$. Using this equation of state, the definition of the specific enthalpy $h=\left(\rho_{0}+\rho_{i}+P\right) / \rho_{0}$, and a thermodynamic relation (Gibbs-Duhem relation)

$$
\frac{d h}{h}=\frac{d P}{\rho_{0}+\rho_{i}+P},
$$

we obtain the internal energy density

$$
\rho_{i}=\frac{\kappa}{\Gamma-1} \rho_{0}^{\Gamma}
$$

Since dimensions enter the problem only through the constant $\kappa$, it is convenient to rescale all dimensional quantities with respect to the polytropic length scale

$$
R_{\text {poly }} \equiv \kappa^{1 /(2 \Gamma-2)}
$$

\section{Boundary conditions}

In order to solve the gravitational field equations (7), (8), and (9), we have to set appropriate boundary conditions on two different boundaries: outer boundaries at spatial infinity and inner boundaries on the black hole horizons.

The boundary conditions at spatial infinity follow from the assumption of asymptotic flatness. With the help of a radial coordinate transformation $u=1 / r$ in the external computational domain our computational grid extends to spatial infinity [41, 45], and we can impose the exact boundary conditions

$$
\begin{aligned}
& \left.\psi\right|_{r \rightarrow \infty}=1, \\
& \left.\beta^{i}\right|_{r \rightarrow \infty}=(\boldsymbol{\Omega} \times \boldsymbol{R})^{i}, \\
& \left.\alpha\right|_{r \rightarrow \infty}=1,
\end{aligned}
$$

where $\Omega$ is the orbital angular velocity of the binary system measured at infinity, and $\boldsymbol{R}=(X, Y, Z)$ is a Cartesian coordinate which origin is located at the center of mass of the binary system. Here, we express the shift vector $\beta^{i}$ in a corotating coordinate system that we adopt throughout our calculation. In an inertial coordinate system, the shift vector would tend to zero at spatial infinity, while in the corotating coordinate system of the numerical code the shift vector diverges at spatial infinity. For computational purposes it is therefore convenient to write the shift vector as a sum of the rotational shift term $\beta_{\text {rot }}^{i} \equiv(\boldsymbol{\Omega} \times \boldsymbol{R})^{i}$ and a residual part (which tends to zero at spatial infinity), and solve the equations only for the latter (see Appendix $\mathrm{A}$ for a detailed explanation of the decomposition of the metric quantities and related equations).

The inner boundary conditions arise from the excision of the black hole interior. The assumption that the black hole is in equilibrium leads to a set of boundary conditions for the conformal factor and shift vector [39] (see 
also [40, 46] as well as the related isolated horizon formalism, e.g. 47, 48]). The boundary condition for the conformal factor is

$$
\left.\tilde{s}^{k} \tilde{\nabla}_{k} \ln \psi\right|_{\mathcal{S}}=-\left.\frac{1}{4}\left(\tilde{h}^{i j} \tilde{\nabla}_{i} \tilde{s}_{j}-\psi^{2} J\right)\right|_{\mathcal{S}}
$$

where $s^{i} \equiv \psi^{-2} \tilde{s}^{i}$ is the outward pointing unit vector normal to the excision surface and $h_{i j}$ is the induced metric on the excision surface, $h_{i j} \equiv \psi^{4} \tilde{h}_{i j}=\gamma_{i j}-s_{i} s_{j}$. The quantity $J$ is computed from the projection of the extrinsic curvature $K_{i j}$ as $J \equiv h^{i j} K_{i j}$. The boundary condition on the normal component of the shift vector is

$$
\left.\beta_{\perp}\right|_{\mathcal{S}}=\left.\alpha\right|_{\mathcal{S}}
$$

while the tangential components have to satisfy

$$
\left.\beta_{\|}^{i}\right|_{\mathcal{S}}=\epsilon_{j k}^{i} \Omega_{r}^{j} x^{k}
$$

Here $\Omega_{r}^{j}$ is the black hole spin angular velocity vector, which we take to be aligned with the $Z$-axis, and $x^{k}$ is a Cartesian coordinate centered on the black hole. To construct approximately nonspinning black holes, we set the shift vector according to Eqs. (27) and (28) with $\Omega_{r}=\Omega$. This assignment corresponds to a "leading-order approximation" in the language of [40], and we plan to implement their more accurate condition for nonspinning black holes in the future. According to 39], the boundary condition on the lapse function can be chosen freely. In this paper, we choose a Neumann boundary condition

$$
\left.\frac{d \alpha}{d r}\right|_{\mathcal{S}}=0
$$

on the excised surface, where $r$ is a radial isotropic coordinate.

\section{E. Orbital angular velocity}

In this paper we take the rotational axis of the binary system to be the $Z$-axis, and the line connecting the black hole and neutron star centers to be the $X$-axis. To impose a quasicircular orbit we require a force balance along the $X$-axis at the center of the neutron star, which results in the condition 41.

$$
\left.\frac{\partial \ln h}{\partial X}\right|_{\left(X_{\mathrm{NS}}, Y_{\mathrm{NS}}, 0\right)}=0 .
$$

Here $X_{\mathrm{NS}}$ and $Y_{\mathrm{NS}}$ denote the $X$ and $Y$-coordinates of the center of the neutron star relative to the rotational axis of the binary system. Imposing this condition determines the orbital angular velocity $\Omega$. We confirm that the orbital angular velocity obtained by this method agrees with that obtained by requiring the enthalpy at two points on the neutron star's surface to be equal [27] within one part in $10^{-6}$.

\section{F. Global quantities}

It is reasonable to require that the irreducible mass of the black hole and the baryon rest mass of the neutron star are conserved during the inspiral of the BHNS binaries. For such a constant-mass sequence we then monitor the Arnowitt-Deser-Misner (ADM) mass, the Komar mass, and the total angular momentum, all of which are defined globally as follows.

The irreducible mass of the black hole is defined as

$$
M_{\mathrm{irr}}^{\mathrm{BH}} \equiv \sqrt{\frac{A_{\mathrm{EH}}}{16 \pi}},
$$

where $A_{\mathrm{EH}}$ is the proper area of the event horizon. In practice we approximate this area with that of the apparent horizon, $A_{\mathrm{AH}}$, which is computed from an integral on the excision surface $\mathcal{S}$,

$$
A_{\mathrm{AH}}=\int_{\mathcal{S}} \psi^{4} d^{2} x
$$

The baryon rest mass of the neutron star is

$$
M_{\mathrm{B}}^{\mathrm{NS}}=\int \rho_{0} u^{t} \sqrt{-g} d^{3} x,
$$

where $g$ is the determinant of $g_{\mu \nu}$. In our case we have $\sqrt{-g}=\alpha \psi^{6}$, so that

$$
M_{\mathrm{B}}^{\mathrm{NS}}=\int \rho_{0} \alpha u^{t} \psi^{6} d^{3} x .
$$

In the polytropic units of Eq. (22) we normalize the baryon rest mass according to

$$
\bar{M}_{\mathrm{B}}^{\mathrm{NS}} \equiv \frac{M_{\mathrm{B}}^{\mathrm{NS}}}{R_{\text {poly }}},
$$

whereby $\bar{M}_{\mathrm{B}}^{\mathrm{NS}}$ is the rest mass of the polytrope for polytropic constant $\kappa=1$.

We express the ADM mass in isotropic Cartesian coordinates as

$$
M_{\mathrm{ADM}}=-\frac{1}{2 \pi} \oint_{\infty} \partial^{i} \psi d S_{i}
$$

The Komar mass can be written as

$$
M_{\text {Komar }}=\frac{1}{4 \pi} \oint_{\infty} \partial^{i} \alpha d S_{i},
$$

where we use the fact that the shift vector falls off sufficiently rapidly. The total angular momentum is

$$
J_{i}=\frac{1}{16 \pi} \epsilon_{i j k} \oint_{\infty}\left(X^{j} K^{k l}-X^{k} K^{j l}\right) d S_{l},
$$

where $X^{i}$ is a spatial Cartesian coordinate relative to the center of mass of the binary system. Finally, the linear momentum is

$$
P^{i}=\frac{1}{8 \pi} \oint_{\infty} K^{i j} d S_{j}
$$


where we have assumed maximal slicing $K=0$.

During the iteration, we require that the linear momentum vanishes. We enforce this by changing the position of the black hole and neutron star relative to the location of the axis of rotation. Stated differently, this condition fixes the location of the axis of rotation.

We define the binding energy of the binary system as

$$
E_{\mathrm{b}}=M_{\mathrm{ADM}}-M_{0},
$$

where $M_{0}$ is the ADM mass of the binary system at infinite orbital separation, as defined by the sum of the irreducible mass of the isolated black hole and the ADM mass of an isolated neutron star with the same baryon rest mass,

$$
M_{0} \equiv M_{\mathrm{irr}}^{\mathrm{BH}}+M_{\mathrm{ADM}, 0}^{\mathrm{NS}} .
$$

In order to measure a global error in the numerical results, we define the virial error as the fractional difference between the ADM mass and Komar mass,

$$
\delta M \equiv\left|\frac{M_{\mathrm{ADM}}-M_{\mathrm{Komar}}}{M_{\mathrm{ADM}}}\right|
$$

\section{NUMERICAL RESULTS}

Our numerical code is based on the spectral method LORENE library routines developed by the Meudon relativity group [49]. Our computational grid depends on the orbital separation, the mass of the neutron star, and the mass ratio. For example, the grid is divided into 10 (8) domains for the black hole (neutron star) at large separations for a neutron star mass of $\bar{M}_{\mathrm{B}}^{\mathrm{NS}}=0.15$ and mass ratio $M_{\mathrm{irr}}^{\mathrm{BH}} / M_{\mathrm{ADM}, 0}^{\mathrm{NS}}=5$. Each domain for the black hole is covered by $N_{r} \times N_{\theta} \times N_{\phi}=41 \times 33 \times 32$ collocation points and those for the neutron star by $25 \times 17 \times 16$, where $N_{r}, N_{\theta}$, and $N_{\phi}$ denote the collocation points in the radial, polar, and azimuthal directions, respectively. We use a larger number of collocation points for the black hole domains than the neutron star domains because the source terms of the gravitational field equations for the black hole vary slightly around the neutron star position. The amplitude of this excess in the source terms is small but significantly affects the accuracy of the results. In order to resolve this source in the black hole domains, which are centered on the black hole, we need a higher angular resolution. On the other hand, the source terms of the gravitational field equations for the neutron star have large contributions only near the neutron star, and drop off monotonically away from the neutron star. Thus a smaller angular resolution is sufficient in the neutron star domains, which are centered on the neutron star. (See Appendix $\mathrm{A}$ for the decomposition of the gravitational field equations into the black hole and neutron star parts.)

As stated above, we choose $\Gamma=2$ for the adiabatic index of the polytropic equation of state in this paper, and compute several different constant-mass inspiral sequences. We consider neutron stars of rest mass
$\bar{M}_{\mathrm{B}}^{\mathrm{NS}}=0.15$ and 0.10 , with corresponding compactions of $M_{\mathrm{ADM}, 0}^{\mathrm{NS}} / R_{0}=0.145$ and 0.0879 . Here $R_{0}$ is the areal radius of the neutron star in isolation. The maximum baryon rest mass for spherical $\Gamma=2$ polytropes in isolation is $\bar{M}_{\mathrm{B}}^{\max }=0.180$. Our more compact polytrope with $\bar{M}_{\mathrm{B}}^{\mathrm{NS}}=0.15$ has a compaction that is reasonably realistic (e.g. it would apply to binaries containing neutron stars with baryon rest mass $1.5 M_{\odot}$ and isolated spherical neutron stars with a maximum baryon rest mass of $\left.1.8 M_{\odot}\right)$; we consider the less compact model for purposes of comparison.

We also consider mass ratios $M_{\mathrm{irr}}^{\mathrm{BH}} / M_{\mathrm{ADM}, 0}^{\mathrm{NS}}=1,2,3$, 5 , and 10. Note again that we fix the irreducible mass of the black hole and the baryon rest mass of the neutron star for the construction of constant-mass sequences. For the definition of the mass ratio, however, we use the ADM mass of a spherical isolated neutron star $M_{\mathrm{ADM}, 0}^{\mathrm{NS}}$, since this turns out to be more convenient for comparisons with third-order post-Newtonian results.

We summarize our results in Tables [V] and $\mathrm{V}$ in Appendix B

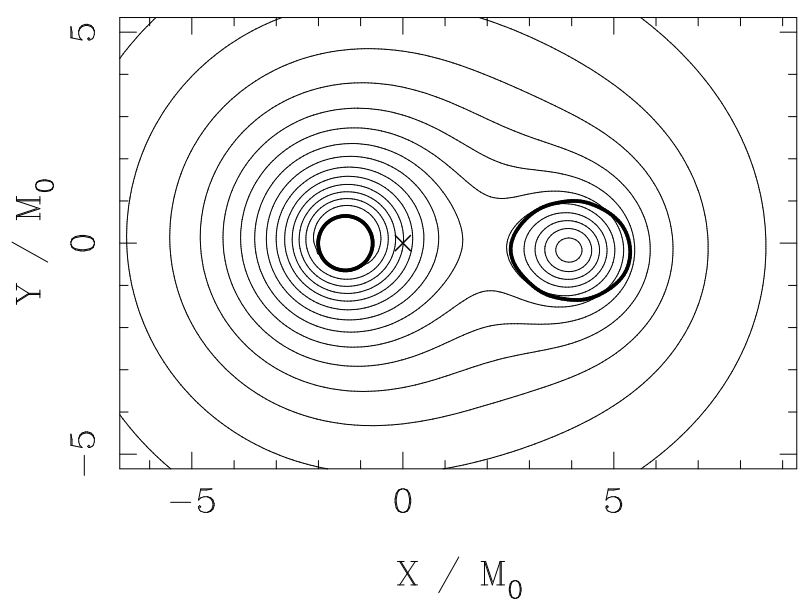

FIG. 1: Contours of the lapse function $\alpha$ in the equatorial plane for our innermost configuration of a binary of mass ratio $M_{\mathrm{irr}}^{\mathrm{BH}} / M_{\mathrm{ADM}, 0}^{\mathrm{NS}}=3$ and neutron star mass $\bar{M}_{\mathrm{B}}^{\mathrm{NS}}=0.15$. The minimum value of the lapse, $\alpha \simeq 0.453$, is located on the excised surface and the maximum value, $\alpha \simeq 0.909$, at the right edge of the figure. The value of the lapse at the point of maximum baryon rest-mass density is about 0.558 . The contour curves are spaced by $\delta \alpha \simeq 0.03$. The cross " $\times$ " indicates the position of the rotation axis.

In Fig. 1 we present contours of the lapse function $\alpha$ for a binary of mass ratio $M_{\mathrm{irr}}^{\mathrm{BH}} / M_{\mathrm{ADM}, 0}^{\mathrm{NS}}=3$ and neutron star mass $\bar{M}_{\mathrm{B}}^{\mathrm{NS}}=0.15$ at the smallest binary separation for which our code converged. The thick solid circle on the left-hand side marks the position of the excised surface (the apparent horizon), while that on the righthand side marks the position of the neutron star surface. The value of the lapse function $\alpha$ on the excised surface is not uniform because we use the Neumann boundary condition (29) rather than a Dirichlet boundary condi- 
tion. The approximate value of the lapse on the excision surface is $\left.\alpha\right|_{\mathcal{S}} \sim 0.45-0.48$.

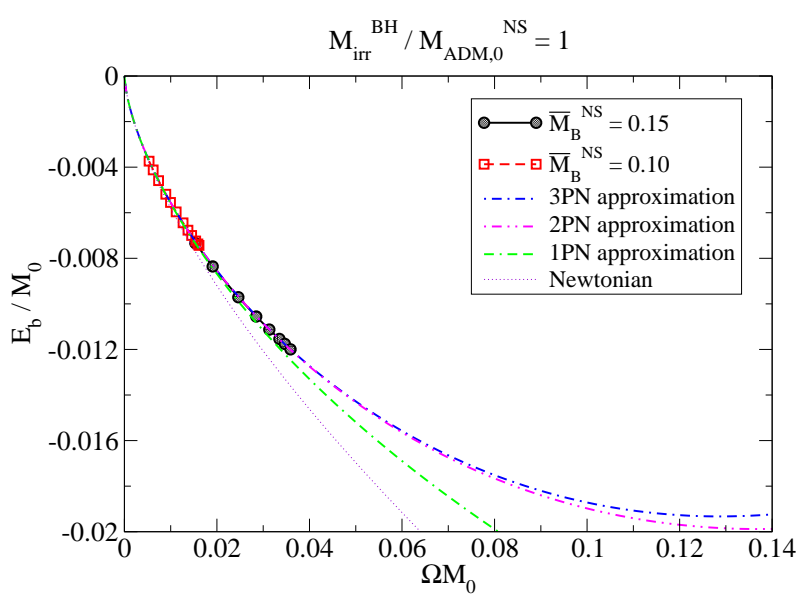

FIG. 2: Fractional binding energy $E_{\mathrm{b}} / M_{0}$ as a function of $\Omega M_{0}$ for binaries of mass ratio $M_{\mathrm{irr}}^{\mathrm{BH}} / M_{\mathrm{ADM}, 0}^{\mathrm{NS}}=1$. The solid line with filled circles shows results for neutron stars of mass $\bar{M}_{\mathrm{B}}^{\mathrm{NS}}=0.15$, and the dashed line with squares for neutron stars of mass of 0.10 . The dash-dotted line denotes the results of the third post-Newtonian approximation [50]. These sequences end due to cusp formation - and hence the onset of tidal disruption - before the binary reaches the ISCO at the turning point of the binding energy.

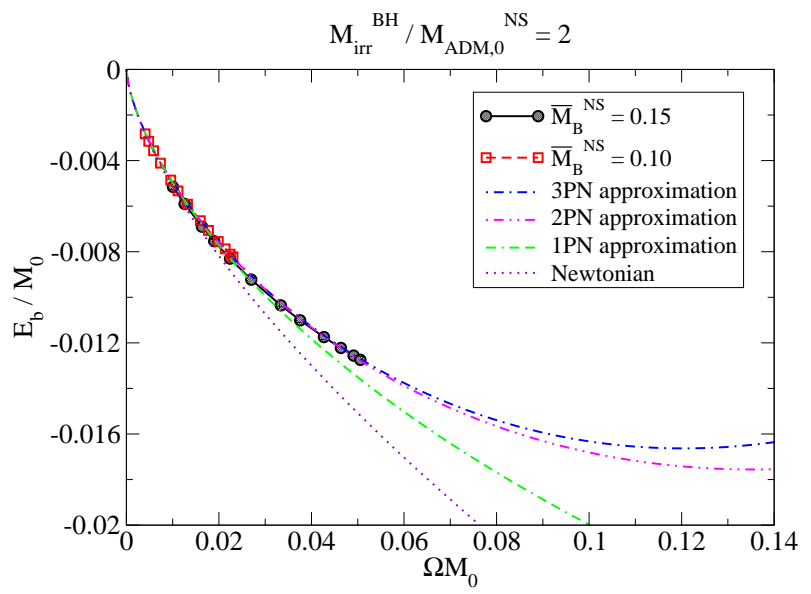

FIG. 3: Same as Fig. 2 but for sequences of mass ratio $M_{\mathrm{irr}}^{\mathrm{BH}} / M_{\mathrm{ADM}, 0}^{\mathrm{NS}}=2$.

In Figs. 2 - 6 we plot the binding energy $E_{\mathrm{b}} / M_{0}$ versus the orbital angular velocity $\Omega M_{0}$ for sequences with mass ratios $1,2,3,5$ and 10 . We find excellent agreement with third-order post-Newtonian approximations [50], especially for large binary separations. For more compact neutron stars with $\bar{M}_{\mathrm{B}}^{\mathrm{NS}}=0.15$, we also find a turning point in the sequences with mass ratios $M_{\mathrm{irr}}^{\mathrm{BH}} / M_{\mathrm{ADM}, 0}^{\mathrm{NS}}=5$ and 10 . Such turning points mark

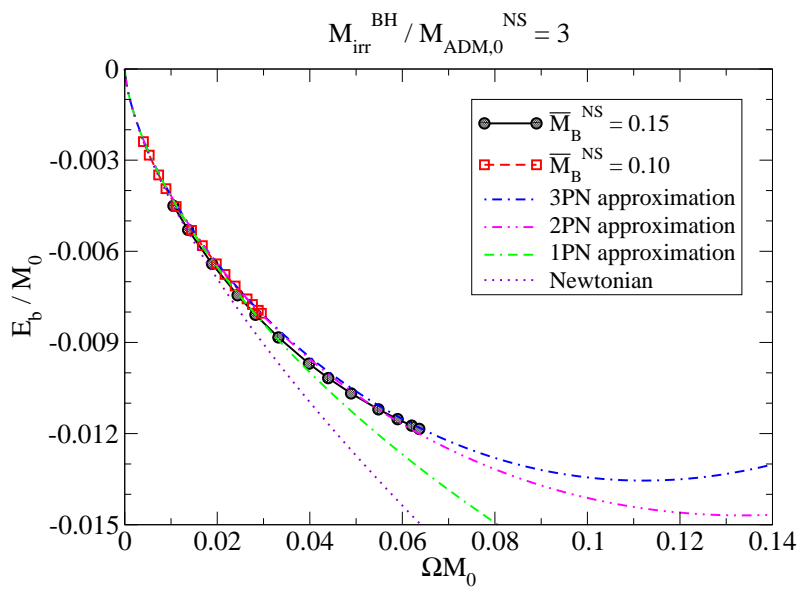

FIG. 4: Same as Fig. 2 but for sequences of mass ratio $M_{\mathrm{irr}}^{\mathrm{BH}} / M_{\mathrm{ADM}, 0}^{\mathrm{NS}}=3$.

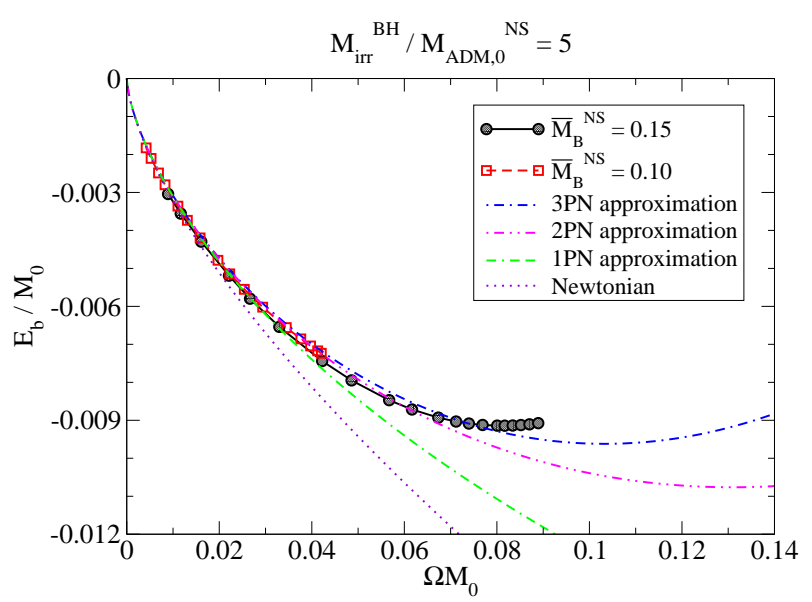

FIG. 5: Same as Fig. 2 but for sequences of mass ratio $M_{\mathrm{irr}}^{\mathrm{BH}} / M_{\mathrm{ADM}, 0}^{\mathrm{NS}}=5$. For the more compact neutron star the binary now encounters an ISCO before the neutron star is tidally disrupted.

the ISCO, inside of which no stable circular orbits can exist. For irrotational binaries this instability is dynamical (see, e.g. [12]). Binaries with larger neutron star compaction and larger mass ratios therefore encounter an ISCO before being tidally disrupted. For all other binaries, the sequences end due to cusp formation - and hence at the onset of tidal disruption - before the binary encounters an ISCO.

Qualitatively, this behavior can be understood very easily from a simple Newtonian analysis. The binary separation at the onset of tidal disruption, $d_{\text {tid }}$, can be estimated by equating the gravitational and tidal forces on a test particle on the surface of the neutron star

$$
\frac{d_{\mathrm{tid}}}{M_{\mathrm{BH}}} \sim\left(\frac{M_{\mathrm{NS}}}{M_{\mathrm{BH}}}\right)^{2 / 3} \frac{R_{\mathrm{NS}}}{M_{\mathrm{NS}}} .
$$




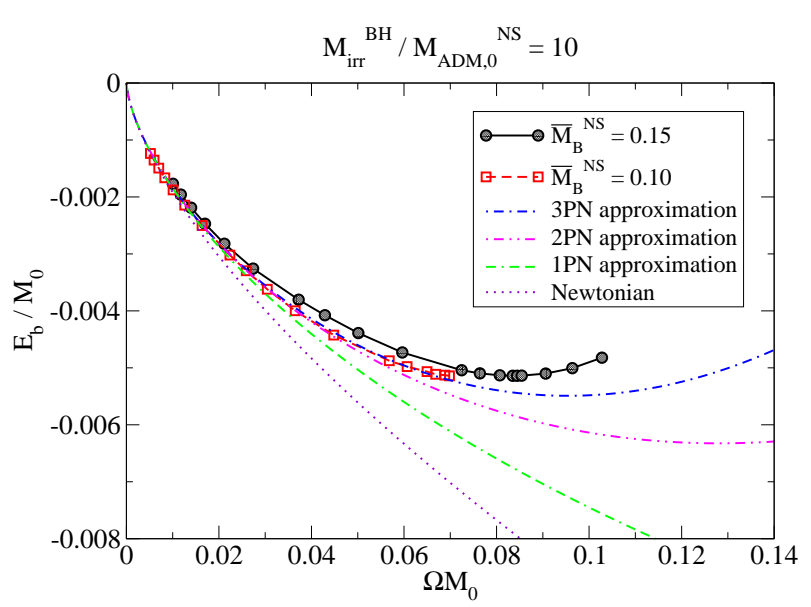

FIG. 6: Same as Fig. 2 but for sequences of mass ratio $M_{\mathrm{irr}}^{\mathrm{BH}} / M_{\mathrm{ADM}, 0}^{\mathrm{NS}}=10$.

If this is smaller than the binary separation at the ISCO, at $d_{\mathrm{ISCO}} / M_{\mathrm{BH}}=6$ for a test particle in Schwarzschild coordinates, the binary encounters the ISCO before being tidally disrupted. This happens for large mass ratios $M_{\mathrm{BH}} / M_{\mathrm{NS}}$ and large compactions $M_{\mathrm{NS}} / R_{\mathrm{NS}}$ - in accordance with our findings.

In the test-particle limit the location of the turning point should coincide with that of test particle orbiting a Schwarzschild black hole of mass $M_{0}, \Omega M_{0}=$ $6^{-3 / 2} \simeq 0.068$. We find similar but slightly larger values, $\Omega M_{0} \sim 0.082$ and $\sim 0.084$ for $M_{\mathrm{irr}}^{\mathrm{BH}} / M_{\mathrm{ADM}, 0}^{\mathrm{NS}}=5$ and 10. Locating the minimum of a numerically generated curve always introduces some additional error, and it is possible that this deviation is entirely a numerical artifact. However, it is also possible that the deviation is a consequence of our "leading-order approximation" of the black hole spin. This approximation leads to a small but non-zero black hole spin, so that effectively we locate the ISCO around a Kerr instead of a Schwarzschild black hole. Further evidence for this hypothesis is the fact that we do not find turning points of the angular momentum that coincide with those of the binding energy. In 39], the authors similarly found that the binding energy and angular momentum for "leading-order" irrotational black hole binaries did not have simultaneous turning points. In [40], however, the authors show that an improved condition for nonspinning black holes leads to a very good agreement between the binding energy and angular momentum turning points. We plan to implement this improved condition in the near future.

As one measure of the error in our numerical calculations we compute the virial expression (42). As shown in Fig. 7, all our configurations have relative virial errors of less than $10^{-3}$, generally on the order of several $\times 10^{-4}$. The errors are smaller at intermediate binary separations, and somewhat larger both at larger binary separations (where the angular resolution becomes worse)
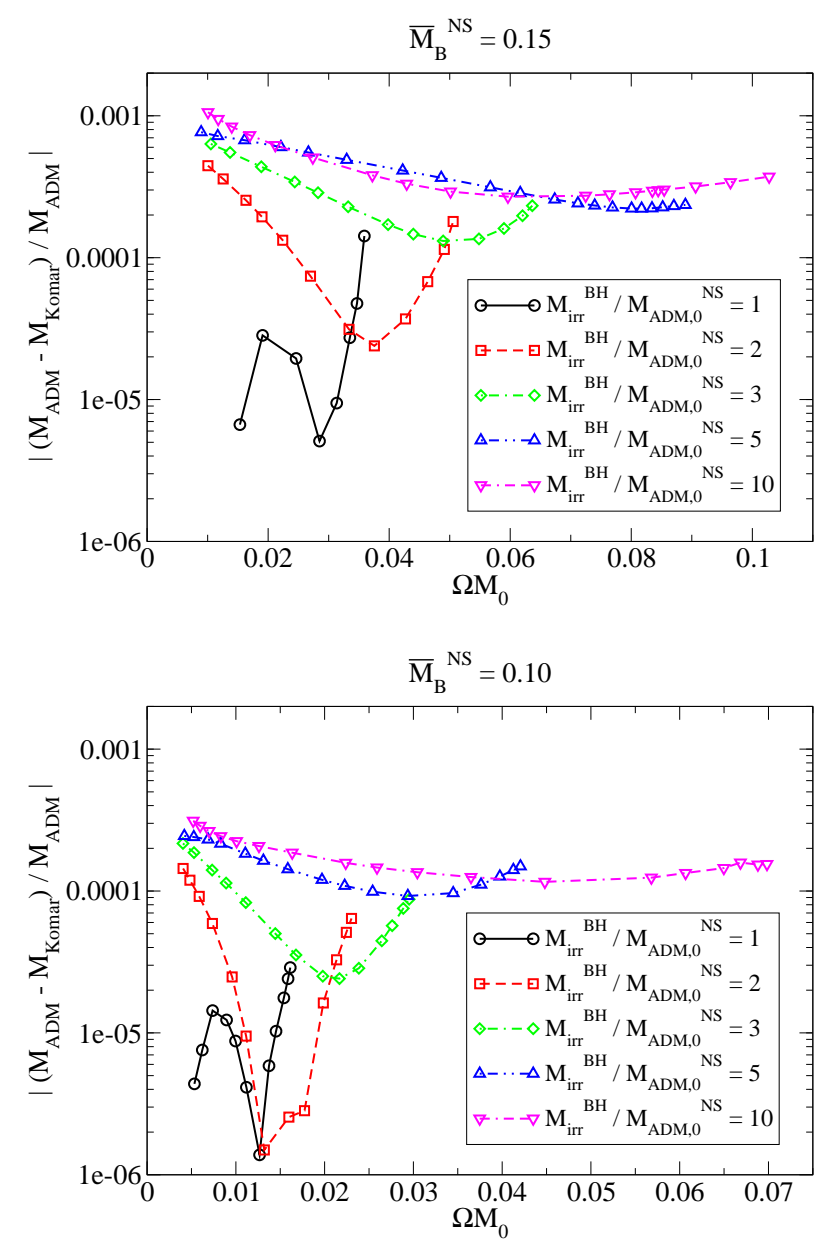

FIG. 7: Virial error $\delta M$ versus $\Omega M_{0}$ for our binary sequences.

and smaller binary separations (where the larger tidal deformation of the neutron star causes larger numerical error).

From the virial error we can estimate the error in the binding energy as follows. We assume that the relative error in the ADM mass is of the same order as the relative virial error,

$$
\left|\frac{M_{\mathrm{ADM}}-M_{\mathrm{ADM}, \text { true }}}{M_{\mathrm{ADM}, \text { true }}}\right| \sim \delta M,
$$

where $M_{\mathrm{ADM} \text {,true }}$ is the true value of the ADM mass. From the definition of the binding energy Eq. (40), we can write the relative difference of the binding energy from its true value $E_{\mathrm{b}, \text { true }}$ as

$$
\left|\frac{E_{\mathrm{b}}-E_{\mathrm{b}, \text { true }}}{E_{\mathrm{b}, \text { true }}}\right| \sim \delta M\left|\frac{M_{\mathrm{ADM}, \text { true }}}{M_{\mathrm{ADM}, \text { true }}-M_{0}}\right| .
$$

Since the term $\left|M_{\mathrm{ADM}, \text { true }} /\left(M_{\mathrm{ADM}, \text { true }}-M_{0}\right)\right|$ on the right-hand side is of order of $10^{2}$ for the binary separations that we calculate, we conclude that the relative error in the binding energy is approximately $10^{2} \delta M$. For 
our least accurate sequences this results in a relative error of several $\times 10^{-2}$ and for our most accurate sequences it is a few $\times 10^{-3}$.
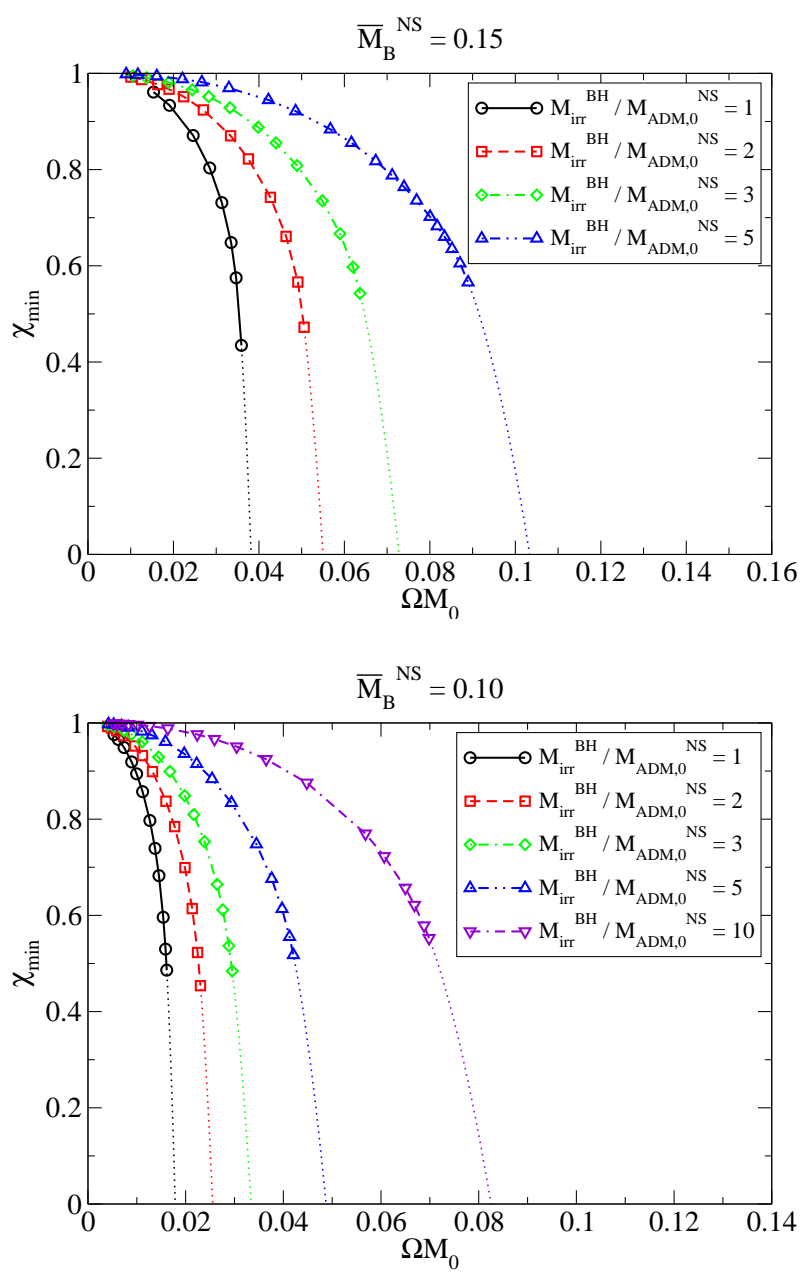

FIG. 8: Minimum of the mass-shedding indicator $\chi_{\min }$ as a function of $\Omega M_{0}$.

TABLE I: Estimated orbital angular velocity at the tidal disruption point.

\begin{tabular}{c|l|l}
\hline \hline & \multicolumn{2}{|c}{$\Omega M_{0}$} \\
$M_{\mathrm{irr}}^{\mathrm{BH}} / M_{\mathrm{ADM}, 0}^{\mathrm{NS}}$ & $\bar{M}_{\mathrm{B}}^{\mathrm{NS}}=0.10$ & $\bar{M}_{\mathrm{B}}^{\mathrm{NS}}=0.15$ \\
\hline 1 & 0.0179 & 0.0382 \\
2 & 0.0255 & 0.0550 \\
3 & 0.0334 & 0.0728 \\
5 & 0.0488 & 0.103 \\
10 & 0.0825 & \\
\hline
\end{tabular}

Using a spectral code we cannot follow our sequences all the way to cusp formation (see our discussion in [28]).
To monitor the formation of a cusp we therefore define a mass-shedding indicator [41]

$$
\chi \equiv \frac{(\partial(\ln h) / \partial r)_{\mathrm{eq}}}{(\partial(\ln h) / \partial r)_{\mathrm{pole}}}
$$

the ratio of the radial derivative of the enthalpy in the equatorial plane at the surface to that to the polar direction at the surface. Given our choice of boundary conditions the minimum point of $\chi$ is not on the $X$ axis (which connects the neutron star with the black hole), but slightly away from the axis. In Fig. 8 we therefore graph the minimum value of $\chi$, searched on the surface of the neutron star, as a function of $\Omega M_{0}$. For spherical stars at infinite separation we have $\chi_{\min }=1$, while $\chi_{\min }=0$ indicates the formation of a cusp and hence tidal breakup. Our spectral code stops converging before reaching $\chi_{\min }=0$, but the sharp drop in $\chi_{\min }$ is an indication of the formation of a cups. Extrapolating to $\chi_{\min }=0$ from the last three points (indicated by the thin dotted lines in Fig. 8) we estimate the orbital angular velocity at the onset of tidal disruption (see Table I). We did not include the sequence with mass ratio $M_{\mathrm{irr}}^{\mathrm{BH}} / M_{\mathrm{ADM}, 0}^{\mathrm{NS}}=10$ and neutron star mass $\bar{M}_{\mathrm{B}}^{\mathrm{NS}}=0.15$ in this analysis, since the binary encounters an ISCO long before the neutron star is tidally disrupted (see also Table IV]. For the sequence with $M_{\mathrm{irr}}^{\mathrm{BH}} / M_{\mathrm{ADM}, 0}^{\mathrm{NS}}=10$ and $\bar{M}_{\mathrm{B}}^{\mathrm{NS}}=0.10$ the extrapolation of $\chi_{\text {min }}$ suggests that tidal disruption occurs at $\Omega M_{0} \simeq 0.0825$ (the last entry in Table I), a value very similar to the values of the ISCO in those two sequences for which we found a turning point. This suggests that the neutron star in this binary may be tidally disrupted just as the orbit becomes unstable.

In [29], where we computed similar BHNS binary sequences but with a Kerr-Schild background, we also considered a binary of mass ratio $M_{\mathrm{irr}}^{\mathrm{BH}} / M_{\mathrm{B}}^{\mathrm{NS}}=5$ and neutron star mass $\bar{M}_{\mathrm{B}}^{\mathrm{NS}}=0.10$. There we estimated that tidal disruption would set in at $\Omega M_{0} \simeq 0.046$. We compute a sequence with the same definition of the mass ratio $M_{\mathrm{irr}}^{\mathrm{BH}} / M_{\mathrm{B}}^{\mathrm{NS}}=5$ for comparison, and find tidal disruption at approximately $\Omega M_{0} \sim 0.053$, a value within $15 \%$ of our earlier value.

As the orbital separation decreases, the maximum density of the neutron star decreases. We define the dimensionless density parameter

$$
q \equiv \frac{P}{\rho_{0}}
$$

and monitor the decrease in the maximum density

$$
\delta q_{\max } \equiv \frac{q_{\max }-q_{\max , 0}}{q_{\max , 0}}
$$

where $q_{\max , 0}$ denotes that of an isolated neutron star with the same baryon rest mass. For $\bar{M}_{\mathrm{B}}^{\mathrm{NS}}=0.15(0.10)$ we have $q_{\max , 0}=0.12665(0.058827)$. Note that $\delta q_{\max }$ is negative for all configurations considered in this study. In Fig. 9 we therefore graph $-\delta q_{\max }$ as a function of the orbital angular velocity $\Omega M_{0}$ on a logarithmic scale. 

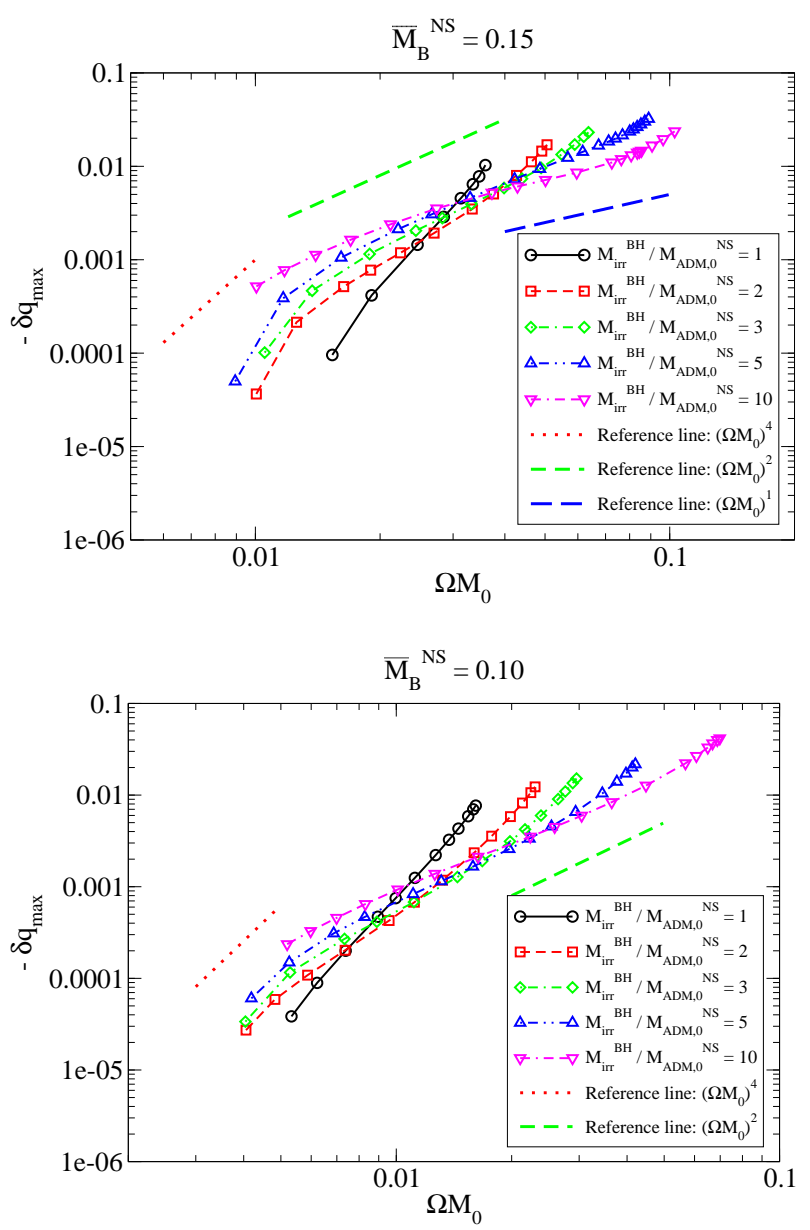

FIG. 9: Decrease in the maximum density parameter $\delta q_{\max }$ as a function of $\Omega M_{0}$.

Within Newtonian gravity, the decrease in the central density scales, to leading order, with

$$
\delta q \propto d^{-\sigma} \propto \Omega^{2 \sigma / 3},
$$

where $d$ is the orbital separation, and for irrotational binaries the index $\sigma$ takes the value $\sigma=6$ (see, e.g. 51] for an analytic derivation and [41] for comparison with numerical results). Here the leading order term is caused by a quadrupole deformation in response to the companion's tidal field. We see from Fig. 9 that the power-law index $\sigma$ is indeed close to 6 for large binary separations. For intermediate binary separations, however, the index is generally smaller, $\sigma \sim 2-3$. For the equal-mass binary $\sigma$ remains close to 6 for the entire sequence, but for increasing mass ratios $\sigma$ decreases at intermediate binary separations. We plan to investigate this behavior with the help of semi-analytic models in a future publication. Finally, for small binary separations, just before tidal disruption sets in, $\sigma$ increases again. This has also been seen for BNS systems [42, 43] and may be caused by higher order multipole deformations.

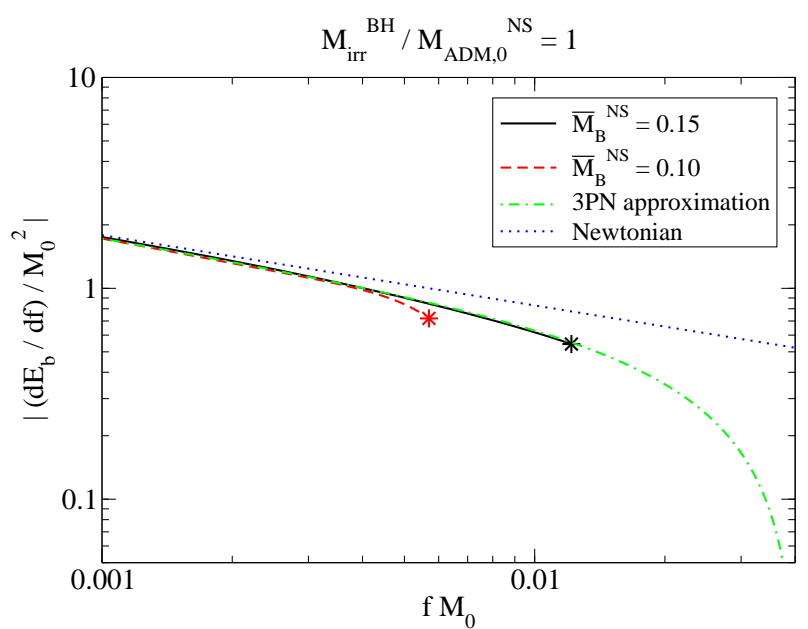

FIG. 10: The energy spectrum $d E_{\mathrm{b}} / d f$ for the sequences with $M_{\mathrm{irr}}^{\mathrm{BH}} / M_{\mathrm{ADM}, 0}^{\mathrm{NS}}=1$ as a function of the dimensionless gravitational wave frequency $f M_{0}$. The solid, dashed, dash-dotted, and dotted curves show the fits to the $\bar{M}_{\mathrm{B}}^{\mathrm{NS}}=0.15$ and $\bar{M}_{\mathrm{B}}^{\mathrm{NS}}=0.1$ sequences and the $3 \mathrm{PN}$ and Newtonian expressions, respectively. Asterisks denote the approximate point where a cusp forms and tidal disruption begins, terminating the sequence, at frequencies given by Table I

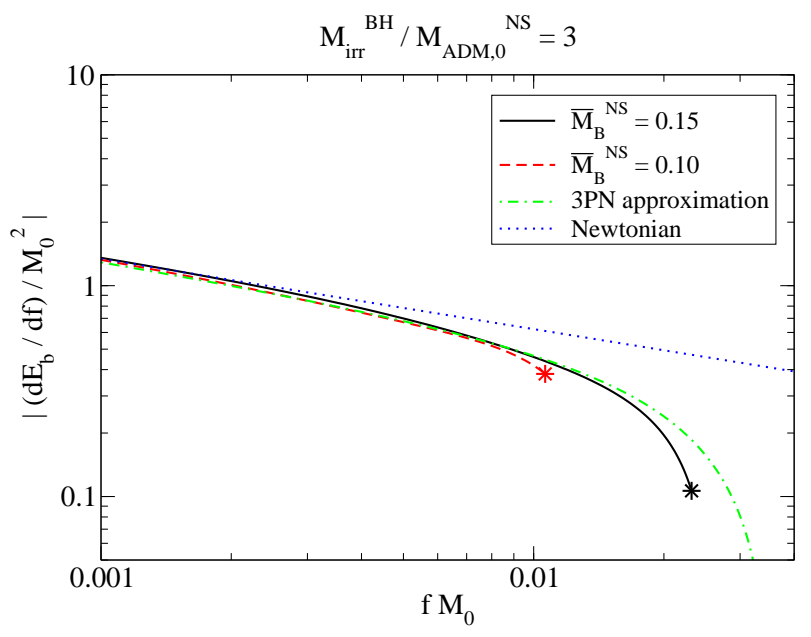

FIG. 11: Same as Fig. 10, but for the sequences with $M_{\mathrm{irr}}^{\mathrm{BH}} / M_{\mathrm{ADM}, 0}^{\mathrm{NS}}=3$.

Finally, we turn our attention to the energy spectrum of gravitational waves emitted from a BHNS binary, which is calculated from the Fourier transform of the gravitational wave strains averaged over all angles (see, e.g. [52]),

$$
\frac{d E}{d f}=\frac{\pi}{2}\left(4 \pi r^{2}\right) f^{2}\left\langle\left|\tilde{h}_{+}(f)\right|^{2}+\left|\tilde{h}_{\times}(f)\right|^{2}\right\rangle,
$$




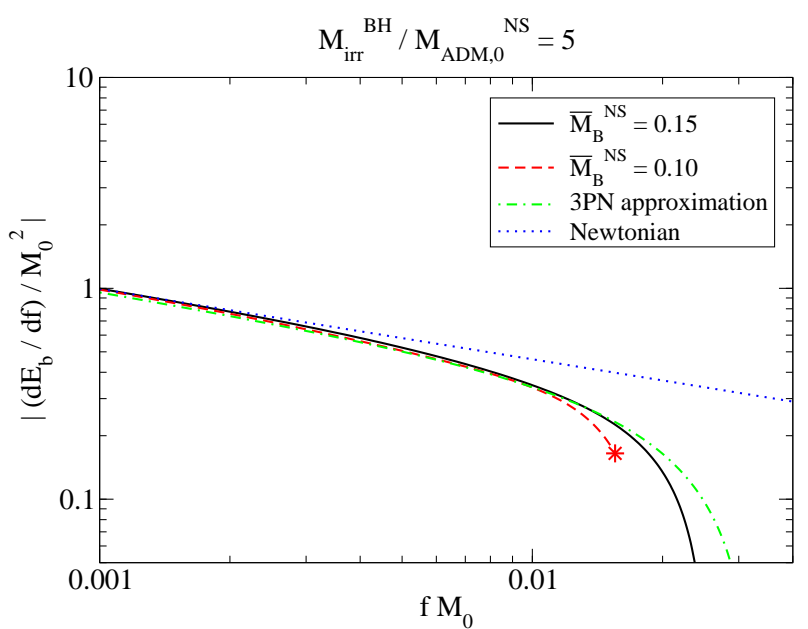

FIG. 12: Same as Fig. 10 but for the sequences with $M_{\mathrm{irr}}^{\mathrm{BH}} / M_{\mathrm{ADM}, 0}^{\mathrm{NS}}=5$. Note that the sequence with $\bar{M}_{\mathrm{B}}^{\mathrm{NS}}=0.15$ reaches a minimum binding energy, where $d E_{\mathrm{b}} / d f=0$, prior to tidal disruption.

where the brackets denote angle averaging and

$$
\tilde{h}(f)=\int_{-\infty}^{\infty} h(t) e^{2 \pi i f t} d t
$$

where $f$ is the gravitational wave frequency, rather than the orbital frequency. From the results of our quasiequilibrium sequences, we can compute approximate gravitational wave energy spectra using the methods described in 53. To do so, we assume that gravitational waves from the binary are emitted coherently with $f=\Omega / \pi$ at any given moment in time, where $\Omega$ is the standard orbital angular velocity, and further assume that the system is adiabatic and infall velocities negligible, so that the gravitational wave energy losses drive the system gradually through a series of quasiequilibrium configurations. Under these assumptions, we recover a simple expression for the gravitational wave energy spectrum,

$$
\frac{d E}{d f} \approx-\pi\left(\frac{d E_{\mathrm{b}}}{d \Omega}\right)_{\mathrm{QE}}
$$

i.e., we can calculate the energy spectrum by numerically differentiating the binding energy along the quasiequilibrium sequence with respect to the angular frequency. This method has been shown to reproduce closely the actual energy spectrum calculated from a fully dynamical binary neutron star merger calculation in the frequency regime of interest [54]. While we can numerically extrapolate up to the frequency at which we find a minimum in the binding energy, representing the ISCO, our results do not apply inside the separation at which a cusp forms and tidal disruption begins (see Table I). Dynamically, this would lead to the beginning of mass transfer and a rapid evolution away from quasiequilibrium, which in turn would produce a qualitatively different gravitational wave signal in both the time and frequency domains.

Instead of taking derivatives of our numerical data for the binding energy directly (see Tables [V] and VD), we first fit these data to a fourth-order polynomial of the form

$$
\frac{E_{\mathrm{b}}}{M_{0}}=a_{\mathrm{N}} x\left(1+a_{1} x+a_{2} x^{2}+a_{3} x^{3}+a_{4} x^{4}\right)
$$

where $x \equiv\left(\Omega M_{0}\right)^{2 / 3}=\left(\pi f M_{0}\right)^{2 / 3}$. Given this parametrization of the polynomial, the coefficients $a_{i}$ can be interpreted as post-Newtonian terms of order $i$. The leading order, Newtonian term, is given by

$$
a_{\mathrm{N}}=-\frac{\nu}{2} \equiv-\frac{M_{\mathrm{irr}}^{\mathrm{BH}} M_{\mathrm{ADM}, 0}^{\mathrm{NS}}}{2 M_{0}^{2}}
$$

Here $\nu$ is the symmetric mass ratio, i.e., the ratio of the reduced mass of the system to the total mass. We tabulate the coefficients $a_{i}$, together with the exact $3 \mathrm{PN}$ coefficients for point-mass systems as listed in [50], for the different binary sequences in Table II. We also include the "cutoff frequencies" $f_{n}$, denoting the frequency at which the spectral energy is $n \%$ of its Newtonian counterpart,

$$
\begin{aligned}
\frac{1}{M_{0}^{2}} \frac{d E_{\mathrm{b}}}{d f}\left(f_{n}\right) & =\frac{n}{100} \times\left(\frac{1}{M_{0}^{2}} \frac{d E_{\mathrm{b}}}{d f}\right)_{\mathrm{N}} \\
& =\frac{n}{100} \frac{2 \pi a_{\mathrm{N}}}{3}\left(\pi f_{n} M_{0}\right)^{-1 / 3},
\end{aligned}
$$

for $n=75,50$ and 25 .

In Figs. 10 - 12, we show the results of the energy spectrum of gravitational waves. As is expected, binaries of comparable mass terminate with the formation of a cusp, rather than at an ISCO denoting a minimum in the binding energy of the binary. For the smallest black hole mass we consider, $M_{\mathrm{irr}}^{\mathrm{BH}} / M_{\mathrm{ADM}, 0}^{\mathrm{NS}}=1$, shown in Fig. 10, tidal disruption occurs before we see significant deviations from either the lowest-order Newtonian point-mass results or the exact $3 \mathrm{PN}$ point-mass expression. As the black hole mass and tidal disruption frequency both increase, so do the deviations in the gravitational wave energy spectrum away from the Newtonian point-mass result. This is especially true for the more compact neutron star models with $\bar{M}_{\mathrm{B}}^{\mathrm{NS}}=0.15$. For a mass ratio $M_{\mathrm{irr}}^{\mathrm{BH}} / M_{\mathrm{ADM}, 0}^{\mathrm{NS}}=5$, shown in Fig. 12, the less compact neutron star model with $\bar{M}_{\mathrm{B}}^{\mathrm{NS}}=0.1$ (dashed curve) shows significant deviations from the $3 \mathrm{PN}$ result at the tidal disruption point, whereas the more compact model with $\bar{M}_{\mathrm{B}}^{\mathrm{NS}}=0.15$ (solid curve) does reach the ISCO before the onset of tidal disruption. This is also the case for the most extreme mass ratio we consider, $M_{\mathrm{irr}}^{\mathrm{BH}} / M_{\mathrm{ADM}, 0}^{\mathrm{NS}}=10$, for which any physically reasonably compact neutron star model reaches an ISCO prior to the tidal disruption point, as had been confirmed by our previous dynamical calculations [7].

We note that the gravitational wave spectrum should produce an important constraint on the neutron star 
compaction, regardless of how the sequence terminates. As the compaction decreases, tidal effects grow in importance, and tend to increases the binding energy of the system (lowering the magnitude of the negative binding energy), especially at smaller separations, implying that the gravitational wave spectrum will be cutoff at lower frequencies.

TABLE II: Exact 3PN data from [50], along with polynomial fit results from the sequences with neutron star masses $\bar{M}_{\mathrm{B}}^{\mathrm{NS}}=0.15$ and 0.10. Coefficients are defined by Eq. (53). Characteristic gravitational wave cutoff frequencies $f_{75} M_{0}$, $f_{50} M_{0}$, and $f_{25} M_{0}$ are defined by Eq. (55). Cutoff frequencies shown in boldface are interpolated from our data, while those in regular text are extrapolated. Values shown in italics occur at separations within our best estimate of the tidal disruption point, given by the values in Table \. and likely overestimate the true cutoff frequency.

\begin{tabular}{l|cccc|ccc}
\hline \hline & $a_{1}$ & $a_{2}$ & $a_{3}$ & $a_{4}$ & $f_{75} M_{0}$ & $f_{50} M_{0}$ & $f_{25} M_{0}$ \\
\hline \hline \multicolumn{7}{c}{$M_{\mathrm{irr}}^{\mathrm{BH}} / M_{\mathrm{ADM}, 0}^{\mathrm{NS}}=1$} \\
\hline $3 \mathrm{PN}$ & -0.771 & -2.78 & -0.967 & & $1.05(-2)$ & $2.14(-2)$ & $3.15(-2)$ \\
0.15 & 0.155 & -23.6 & 156. & -401. & $\mathbf{9 . 8 0}(-3)$ & $1.96(-2)$ & $2.49(-2)$ \\
0.10 & 1.42 & -128. & $2.45(3)$ & $-1.66(4)$ & $5.48(-3)$ & $6.81(-3)$ & $7.64(-3)$ \\
\hline \multicolumn{7}{c}{$M_{\mathrm{irr}}^{\mathrm{BH}} / M_{\mathrm{ADM}, 0}^{\mathrm{NS}}=2$} \\
\hline $3 \mathrm{PN}$ & -0.769 & -2.85 & -2.02 & $1.02(-2)$ & $2.05(-2)$ & $2.98(-2)$ \\
0.15 & 1.66 & -54.6 & 386. & $-1.02(3)$ & $\mathbf{9 . 2 2}(-3)$ & $1.76(-2)$ & $2.15(-2)$ \\
0.10 & 1.77 & -105. & $1.43(3)$ & $-6.94(3)$ & $7.48(-3)$ & $9.72(-3)$ & $1.10(-2)$ \\
\hline \multicolumn{7}{c}{$M_{\mathrm{irr}}^{\mathrm{BH}} / M_{\mathrm{ADM}, 0}^{\mathrm{NS}}=3$} \\
\hline $3 \mathrm{PN}$ & -0.766 & -2.93 & -3.34 & $9.89(-3)$ & $1.95(-2)$ & $2.80(-2)$ \\
0.15 & 1.28 & -37.0 & 210. & -494. & $\mathbf{9 . 5 7}(-3)$ & $\mathbf{1 . 7 5}(-2)$ & $2.28(-2)$ \\
0.10 & 1.47 & -74.2 & 815. & $-3.33(3)$ & $\mathbf{8 . 5 7}(-3)$ & $1.20(-2)$ & $1.38(-2)$ \\
\hline \multicolumn{7}{c}{$M_{\mathrm{irr}}^{\mathrm{BH}} / M_{\mathrm{ADM}, 0}^{\mathrm{NS}}=5$} \\
\hline $3 \mathrm{PN}$ & -0.762 & -3.05 & -5.20 & $9.51(-3)$ & $1.84(-2)$ & $2.60(-2)$ \\
0.15 & 0.802 & -26.1 & 138. & -357. & $\mathbf{1 . 0 1}(-2)$ & $\mathbf{1 . 7 2 ( - 2 )}$ & $\mathbf{2 . 2 1}(-2)$ \\
0.10 & 1.03 & -47.7 & 423. & $-1.47(3)$ & $\mathbf{9 . 5 4 ( - 3 )}$ & $1.45(-2)$ & $1.71(-2)$ \\
\hline \multicolumn{7}{c}{$M_{\mathrm{irr}}^{\mathrm{BH}} / M_{\mathrm{ADM}, 0}^{\mathrm{NS}}=10$} \\
\hline $3 \mathrm{PN}$ & -0.757 & -3.18 & -7.36 & $9.14(-3)$ & $1.73(-2)$ & $2.42(-2)$ \\
0.15 & -0.934 & -14.7 & 122. & -365. & $\mathbf{6 . 9 4}(-3)$ & $\mathbf{1 . 7 1}(-2)$ & $\mathbf{2 . 3 0}(-2)$ \\
0.10 & 0.305 & -28.5 & 212. & -643. & $\mathbf{9 . 3 5 ( - 3 )}$ & $\mathbf{1 . 6 7}(-2)$ & $\mathbf{2 . 0 7}(-2)$ \\
\hline \multicolumn{7}{c}{}
\end{tabular}

\section{COMPARISON WITH PREVIOUS RESULTS}

We can compare our numerical results with those from previous attempts to model BHNS binaries. In particular, we compare with our previous work that assumed extreme mass ratios [28], our comparable-mass results for a Kerr-Schild background [29], and the results of [30] for comparable masses and a spatially flat background, as in this study.

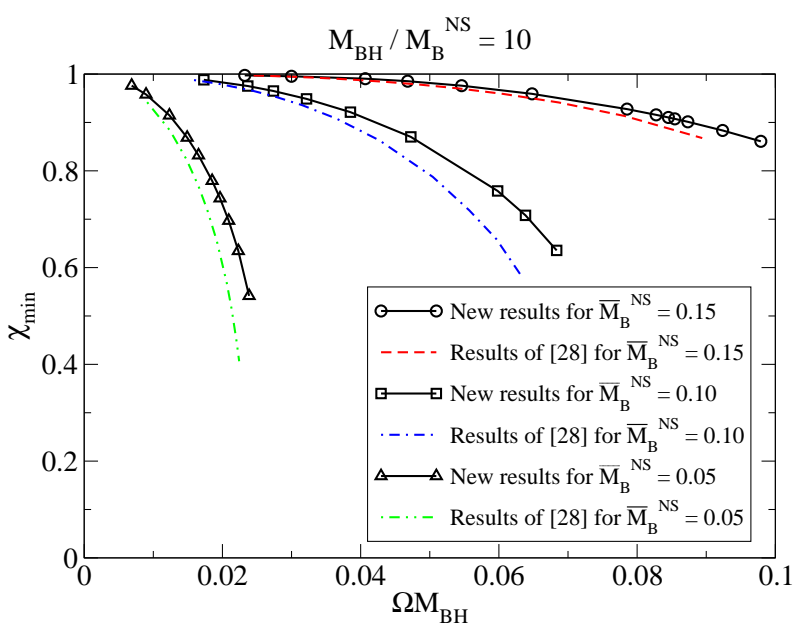

FIG. 13: Comparison of our new results to those calculated assuming an extreme mass ratio for a spatially flat background geometry [28] for the minimum of the mass-shedding indicator $\chi_{\text {min }}$. Here $M_{\mathrm{BH}}$ denotes either the black hole irreducible mass (for our new results) or the background mass (for the results of [28]); see text.

\section{A. Comparison with extreme mass ratio results}

In Fig. 13, we compare our results for a mass ratio $M_{\mathrm{irr}}^{\mathrm{BH}} / M_{\mathrm{B}}^{\mathrm{NS}}=10$ with those for $M_{\mathrm{BH}} / M_{\mathrm{B}}^{\mathrm{NS}}=10$ in [28] for a spatially flat background, but under the assumption of an extreme mass ratio. Since the tidal effects of the neutron star on the black hole are neglected in the extreme mass-ratio approximation, it is impossible to evaluate the black hole's irreducible mass. In [28], therefore, we instead fixed the background mass of the black hole $M_{\mathrm{BH}}$. Since the difference between $M_{\mathrm{irr}}^{\mathrm{BH}}$ and $M_{\mathrm{BH}}$ scales with the binary's binding energy (see [55] for an analytic, leading-order treatment), the relative difference is at most a few percent for this mass ratio.

Our present results agree well with those calculated assuming an extreme mass ratio. The difference in the orbital angular velocity for the same $\chi_{\min }$ is order $10 \%$. We can explain this $10 \%$ discrepancy as follows: for a given value of $\chi_{\min }$, the tidal deformation of the neutron star by the black hole is approximately the same in the two cases. This implies that the orbital separation is very similar as well. However, the orbital angular velocity in our calculation here is given in terms of the distance from the center of mass of the binary system to the center of the neutron star, while for an extreme mass ratio it is given by the binary separation. This is because with the extreme mass-ratio assumption, we assumed that the binary's center of mass is located at the center of the black hole. The difference between these two definitions of distance, along with a change in the black hole mass we use in the definition of the mass ratio, accounts for the discrepancy in the orbital angular velocities. To Newto- 
nian order, there is a difference between $\Omega=\sqrt{M_{0} / d^{3}}$ in our calculation here and $\Omega=\sqrt{M_{\mathrm{BH}} / d^{3}}$ for the extreme mass ratio. The relative difference between these angular velocities is about $10 \%$ for the mass ratio in question.

\section{B. Comparison with Kerr-Schild coordinates results}

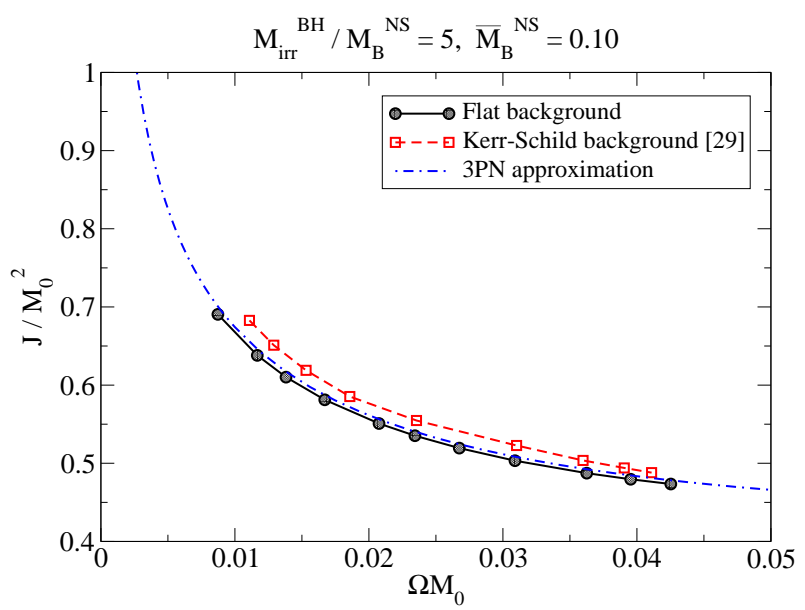

FIG. 14: Comparison of our new results to those calculated using a Kerr-Schild background [29] for the total angular momentum. The dash-dotted line denotes the results of the third post-Newtonian approximation [50].

In Fig. 14 we compare our new results for the total angular momentum with those in 29], for a mass ratio $M_{\mathrm{irr}}^{\mathrm{BH}} / M_{\mathrm{B}}^{\mathrm{NS}}=5$ and neutron star mass $\bar{M}_{\mathrm{B}}^{\mathrm{NS}}=0.10$. The difference between our treatment here and that in 29] is the choice of the spatial background metric $\tilde{\gamma}_{i j}$. In this paper, we assume a flat background, $\tilde{\gamma}_{i j}=\eta_{i j}$, while in 29] we chose a Kerr-Schild metric. We see from Fig. 14 that the behavior of the sequence agrees to within $5 \%$ [56] throughout most of the sequence.

\section{Comparison with previous flat background results}

In [30] (hereafter G06), Grandclément computed similar sequences of BHNS binaries, adopting an approach that in some ways is similar to ours. Both codes are based on the LORENE spectral method library, but are otherwise completely independent. In particular, we impose different inner boundary conditions on the black hole's excision surface, use different conditions to make the black hole nonspinning, and adopt a different decomposition of the equations and variables. We summarize these differences between our implementations in Table III.
TABLE III: Comparison between our implementation and that of G06. Here "Ex. BC" stands for the excision boundary condition, and "Dec. of (7) - (9)" stands for the decomposition of the relevant equations and variables.

\begin{tabular}{l|c|c}
\hline \hline & Here & G06 \\
\hline$\tilde{\gamma}_{i j}$ & $\eta_{i j}$ & $\eta_{i j}$ \\
Ex. BC: $\psi, \beta^{i}$ & Cook \& Pfeiffer [39] & Gourgoulhon et.al. [57] \\
Ex. BC: $\alpha$ & $d \alpha /\left.d r\right|_{\mathcal{S}}=0$ & $\left.\alpha\right|_{\mathcal{S}}=0$ \\
$\Omega_{r}$ of BH & Cook \& Pfeiffer [39] & Caudill et.al. [40] \\
Dec. of (7)-(9) & See Appendix[A] & Gourgoulhon et.al. [57] \\
\hline
\end{tabular}

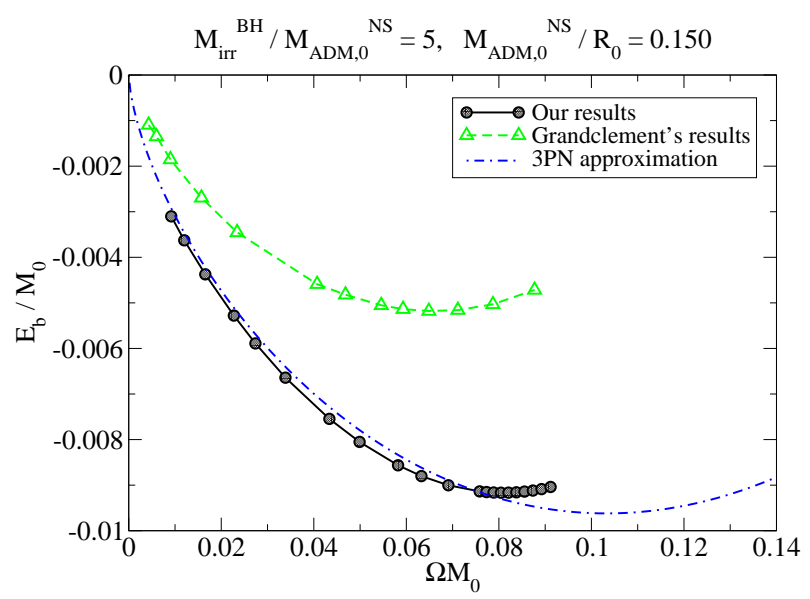

FIG. 15: Comparison between our results and those of G06 for the binding energy as a function of $\Omega M_{0}$, for a binary of mass ratio $M_{\mathrm{irr}}^{\mathrm{BH}} / M_{\mathrm{ADM}, 0}^{\mathrm{NS}}=5$. The solid line with filled circles marks our results for a neutron star compaction of $M_{\mathrm{ADM}, 0}^{\mathrm{NS}} / R_{0}=0.150$, which corresponds to the baryon rest mass of $\bar{M}_{\mathrm{B}}^{\mathrm{NS}}=0.153$. The dashed line with triangles represents those of G06 for a neutron star compaction of 0.150 . The dash-dotted line denotes the results of the third postNewtonian approximation [50].

To compare our results with those of G06, we show in Fig. [15 the binding energy as a function of $\Omega M_{0}$ for two sequences with the same physical parameters. Both sequences are for a binary with mass ratio $M_{\mathrm{irr}}^{\mathrm{BH}} / M_{\mathrm{ADM}, 0}^{\mathrm{NS}}=$ 5 . Our results are for a neutron star rest mass of $\bar{M}_{\mathrm{B}}^{\mathrm{NS}}=0.153$, which corresponds to a compaction of $M_{\mathrm{ADM}, 0}^{\mathrm{NS}} / R_{0}=0.150$. We find agreement with the thirdorder post-Newtonian result to within $3 \%$ except for configurations inside the turning point, and hence inside the ISCO. We also include the binding energy for a neutron star compaction of 0.150 as computed by G06 (and as found in Fig. 3 of G06). Clearly this curve shows a much larger deviation from both our results and the postNewtonian ones, by about a factor of two. We speculate that this deviation could be caused by the larger numerical error in the calculations of G06. According to G06, 
the relative virial error is as large as $2 \%$. If the masses themselves carry an error of $2 \%$, the binding energy may have an error as large as $100 \%$ or more, as we have outlined in the paragraph including Eqs. (44) and (45). This is consistent with the deviations found in Fig. 15.

\section{SUMMARY}

We construct quasiequilibrium sequences of BHNS binaries in general relativity. We solve the constraint equations of general relativity, decomposed in the conformal thin-sandwich formalism and subject to the black hole boundary conditions of [39], together with the relativistic equations of hydrostatic equilibrium. In contrast to our earlier approach in [29], where we solved these equations for a Kerr-Schild background, we now adopt a spatially flat background. We also employ a new decomposition of the equations and variables that leads to a significant improvement in the numerical accuracy.

We construct constant-mass sequences of mass ratios $M_{\mathrm{irr}}^{\mathrm{BH}} / M_{\mathrm{ADM}, 0}^{\mathrm{NS}}=1,2,3,5$, and 10 for neutron star baryon rest masses of $\bar{M}_{\mathrm{B}}^{\mathrm{NS}}=0.15$ and 0.10 . We find excellent agreement between our calculated binding energies and the third-order post-Newtonian approximation, especially for large binary separations, but also agreeing to within $5 \%$ at small separations. We locate the onset of tidal disruption and, for large mass ratios and neutron star compactions, the ISCO.

We find some evidence that our "leading-order approximation" for constructing nonspinning black holes leads to small errors, and plan to implement an improved condition in the near future.

\section{ACKNOWLEDGMENTS}

J.A.F. gratefully acknowledges support through NSF Grant AST-0401533. This paper was supported in part by NSF Grants PHY-0205155 and PHY-0345151 as well as NASA Grant NNG04GK54G to University of Illinois at Urbana-Champaign, and NSF Grant PHY-0456917 to Bowdoin College.

\section{APPENDIX A: DECOMPOSITION OF THE GRAVITATIONAL FIELD VARIABLES}

For the construction of binary configurations with codes that are based on spherical polar coordinates it is natural to use two computational domains, each one centered on one of the binary companions. An equation of the form

$$
\underline{\Delta} \phi=\mathrm{RHS}
$$

can then be split into two equations

$$
\underline{\Delta} \phi_{\mathrm{BH}}=\mathrm{RHS}_{\mathrm{BH}}
$$

$$
\underline{\Delta} \phi_{\mathrm{NS}}=\mathrm{RHS}_{\mathrm{NS}} \text {, }
$$

where $\phi=\phi_{\mathrm{BH}}+\phi_{\mathrm{NS}}$ and RHS $=\mathrm{RHS}_{\mathrm{BH}}+\mathrm{RHS}_{\mathrm{NS}}$. The two equations (A2) and (A3) can then be solved on two computational domains, one centered on the black hole and the other centered on the neutron star.

Clearly, the separation of the source terms RHS into its two parts $\mathrm{RHS}_{\mathrm{BH}}$ and $\mathrm{RHS}_{\mathrm{NS}}$ is not unique. One guiding principle is to move those parts of RHS that are large in the neighborhood of the black hole into $\mathrm{RHS}_{\mathrm{BH}}$, and likewise for the neutron star. Another principle is that each of the source terms should asymptotically coincide with that for the isolated black hole and neutron star when the orbital separation is large. In addition, we use this decomposition freedom to deal with the fact that metric quantities are not defined inside the excised parts of the black hole. It is not clear how to treat such functions if they appear in $\mathrm{RHS}_{\mathrm{NS}}$. One approach is to artificially fill the resulting "hole" in the function with a smooth function (see, e.g. [57]). Instead, we carefully separate the source terms in such a way that no metric quantities that are excised in the black hole interior affect the right-hand side of the neutron star equation.

Specifically, we first decompose the metric quantities as

$$
\begin{aligned}
& \alpha=\alpha_{\mathrm{BH}}+\alpha_{\mathrm{NS}}, \\
& \beta^{i}=\beta_{\mathrm{BH}}^{i}+\beta_{\mathrm{NS}}^{i}+\beta_{\mathrm{rot}}^{i}, \\
& \psi=\psi_{\mathrm{BH}}+\psi_{\mathrm{NS}},
\end{aligned}
$$

where $\beta_{\text {rot }}^{i}$ is the rotational shift vector. We then decompose the gravitational field equations as follows. Equation (7) becomes

$$
\begin{aligned}
& \underline{\Delta} \psi_{\mathrm{BH}}=-\frac{1}{8} \psi^{-7} \tilde{A}_{i j} \tilde{A}^{i j} \\
& \quad+\frac{1}{8}\left(\psi_{\mathrm{NS}}+c_{\psi}\right)^{-7} \tilde{A}_{i j}^{\mathrm{NS}} \tilde{A}_{\mathrm{NS}}^{i j} \\
& \underline{\Delta} \psi_{\mathrm{NS}}=-2 \pi \psi^{5} \rho-\frac{1}{8}\left(\psi_{\mathrm{NS}}+c_{\psi}\right)^{-7} \tilde{A}_{i j}^{\mathrm{NS}} \tilde{A}_{\mathrm{NS}}^{i j},
\end{aligned}
$$

Eq. (8) is decomposed as

$$
\begin{gathered}
\Delta \beta_{\mathrm{BH}}^{i}+\frac{1}{3} \partial^{i}\left(\partial_{j} \beta_{\mathrm{BH}}^{j}\right)=2 \tilde{A}^{i j} \partial_{j}\left(\alpha \psi^{-6}\right) \\
-2 \tilde{A}_{\mathrm{NS}}^{i j} \partial_{j}\left[\left(\alpha_{\mathrm{NS}}+c_{\alpha}\right)\left(\psi_{\mathrm{NS}}+c_{\psi}\right)^{-6}\right], \\
\Delta \beta_{\mathrm{NS}}^{i}+\frac{1}{3} \partial^{i}\left(\partial_{j} \beta_{\mathrm{NS}}^{j}\right)=16 \pi \alpha \psi^{4} j^{i} \\
+2 \tilde{A}_{\mathrm{NS}}^{i j} \partial_{j}\left[\left(\alpha_{\mathrm{NS}}+c_{\alpha}\right)\left(\psi_{\mathrm{NS}}+c_{\psi}\right)^{-6}\right],
\end{gathered}
$$

and Eq. (9) is written as

$$
\begin{gathered}
\triangleq \alpha_{\mathrm{BH}}=\alpha \psi^{-8} \tilde{A}_{i j} \tilde{A}^{i j}-2 \eta^{i j} \partial_{i} \alpha \partial_{j} \ln \psi \\
-\left(\alpha_{\mathrm{NS}}+c_{\alpha}\right)\left(\psi_{\mathrm{NS}}+c_{\psi}\right)^{-8} \tilde{A}_{i j}^{\mathrm{NS}} \tilde{A}_{\mathrm{NS}}^{i j} \\
\quad+2 \eta^{i j} \partial_{i}\left(\alpha_{\mathrm{NS}}+c_{\alpha}\right) \partial_{j} \ln \left(\psi_{\mathrm{NS}}+c_{\psi}\right), \\
\underline{\Delta} \alpha_{\mathrm{NS}}=4 \pi \alpha \psi^{4}(\rho+S) \\
\quad+\left(\alpha_{\mathrm{NS}}+c_{\alpha}\right)\left(\psi_{\mathrm{NS}}+c_{\psi}\right)^{-8} \tilde{A}_{i j}^{\mathrm{NS}} \tilde{A}_{\mathrm{NS}}^{i j} \\
-2 \eta^{i j} \partial_{i}\left(\alpha_{\mathrm{NS}}+c_{\alpha}\right) \partial_{j} \ln \left(\psi_{\mathrm{NS}}+c_{\psi}\right),
\end{gathered}
$$


where we define $\bar{A}_{\mathrm{NS}}^{i j}$ as

$$
\tilde{A}_{\mathrm{NS}}^{i j} \equiv \frac{\left(\psi_{\mathrm{NS}}+c_{\psi}\right)^{6}}{2\left(\alpha_{\mathrm{NS}}+c_{\alpha}\right)}\left(\partial^{i} \beta_{\mathrm{NS}}^{j}+\partial^{j} \beta_{\mathrm{NS}}^{i}-\frac{2}{3} \eta^{i j} \partial_{k} \beta_{\mathrm{NS}}^{k}\right)
$$

Note that the neutron star equations (A8), (A10), and (A12) contain metric quantities like $\alpha$ and $\psi$ that are not defined inside the excised black hole, but only in products with matter quantities that vanish outside the neutron star. As a consequence, the neutron star equations are regular everywhere. All singular, or un-defined terms have been moved to the black hole equations, where they are harmless since they are excised from the computational grid.

We finally point out that the outer boundary conditions of the total lapse function and total conformal factor are asymptotically flat, i.e., $\left.\alpha\right|_{r \rightarrow \infty}=1$ and $\left.\psi\right|_{r \rightarrow \infty}=1$. This implies that each part of the metric quantities, i.e., $\alpha_{\mathrm{BH}}, \alpha_{\mathrm{NS}}, \psi_{\mathrm{BH}}$, and $\psi_{\mathrm{NS}}$, cannot go to unity individually at infinity. We set the outer boundary conditions for these quantities as $\left.\alpha_{\mathrm{BH}}\right|_{r \rightarrow \infty}=0.5$, $\left.\alpha_{\mathrm{NS}}\right|_{r \rightarrow \infty}=0.5,\left.\psi_{\mathrm{BH}}\right|_{r \rightarrow \infty}=0.5$, and $\left.\psi_{\mathrm{NS}}\right|_{r \rightarrow \infty}=0.5$ for the convenience of the computation. We therefore insert constants $c_{\alpha}=0.5$ and $c_{\psi}=0.5$ to ensure that the total quantities take on their proper asymptotic values.

\section{APPENDIX B: TABLES OF SEQUENCES}

We summarize our results in Tables IV and V. In these tables, we tabulate the orbital angular velocity $\Omega$, binding energy $E_{\mathrm{b}}$, total angular momentum $J$, decrease in the maximum density parameter $\delta q_{\max }$, minimum of the mass-shedding indicator $\chi_{\min }$, and fractional difference $\delta M$ between the ADM mass and the Komar mass along a sequence.
[1] S. J. Waldman (LIGO Scientific Collaboration), Class. Quantum Grav. 23, S653 (2006).

[2] H. Lück (GEO600 Collaboration), Class. Quantum Grav. 23, S71 (2006).

[3] M. Ando (TAMA Collabaration), Class. Quantum Grav. 22, S881 (2005).

[4] F. Acernese (VIRGO Collaboration), Class. Quantum Grav. 23, S635 (2006).

[5] E. Barger, in Proceedings of the 16th Annual Astrophysics Conference on Gamma Ray Bursts in the Swift Era, Maryland, edited by S. Holt, N. Gehrels, and J. Nousek, astro-ph/0602004.

[6] M. Shibata and K. Taniguchi, Phys. Rev. D 73, 064027 (2006).

[7] J. A. Faber, T. W. Baumgarte, S. L. Shapiro, and K. Taniguchi, Astrophys. J. 641, L93 (2006).

[8] D. J. Price and S. Rosswog, Science 312, 719 (2006).

[9] R. Oechslin and H.-T. Janka, in Proceedings of the Albert Einstein Century International Conference, Paris, France 2005, edited by J.-M. Alimi and A. Fuzfa, astro-ph/0604562

[10] S. Chandrasekhar, Ellipsoidal Figures of Equilibrium (Yale University Press, New Heaven, CT, 1969).

[11] L. G. Fishbone, Astrophys. J. 185, 43 (1973).

[12] D. Lai, F, A, Rasio, and S. L. Shapiro, Astrophys. J. Suppl. Ser. 88, 205 (1993).

[13] D. Lai and A. G. Wiseman, Phys. Rev. D 54, 3958 (1996).

[14] K. Taniguchi and T. Nakamura, Prog. Theor. Phys. 96, 693 (1996).

[15] M. Shibata, Prog. Theor. Phys. 96, 917 (1996).

[16] K. Uryū and Y. Eriguchi, Mon. Not. R. Astron. Soc. 303, 329 (1999).

[17] P. Wiggins and D. Lai, Astrophys. J. 532, 530 (2000).

[18] M. Ishii, M. Shibata, and Y. Mino, Phys. Rev. D 71, 044017 (2005).

[19] B. Mashhoon, Astrophys. J. 197, 705 (1975).

[20] B. Carter and J.-P. Luminet, Astron. Astrophys. 121, 97 (1983); Mon. Not. R. Astron. Soc. 212, 23 (1985).

[21] J.-A. Marck, Proc. R. Soc. London A 385, 431 (1983).
[22] W. H. Lee and W. Kluźniak, Astrophys. J. 526, 178 (1999); Mon. Not. R. Astron. Soc. 308, 780 (1999).

[23] W. H. Lee, Mon. Not. R. Astron. Soc. 318, 606 (2000); 328, 583 (2001).

[24] S. Rosswog, R. Speith, and G. A. Wynn, Mon. Not. R. Astron. Soc. 351, 1121 (2004).

[25] S. Kobayashi, P. Laguna, E. S. Phinney, and P. Mészáros, Astrophys. J. 615, 855 (2004).

[26] M. Miller, gr-qc/0106017.

[27] T. W. Baumgarte, M. L. Skoge, and S. L. Shapiro, Phys. Rev. D 70, 064040 (2004).

[28] K. Taniguchi, T. W. Baumgarte, J. A. Faber, and S. L. Shapiro, Phys. Rev. D 72, 044008 (2005).

[29] K. Taniguchi, T. W. Baumgarte, J. A. Faber, and S. L. Shapiro, Phys. Rev. D 74, 041502(R) (2006).

[30] P. Grandclément, Phys. Rev. D. 74, 124002 (2006).

[31] J. A. Faber, T. W. Baumgarte, S. L. Shapiro, K. Taniguchi, and F. A. Rasio, Phys. Rev. D 73, 024012 (2006).

[32] C. F. Sopuerta, U. Sperhake, and P. Laguna, Class. Quantum Grav. 23, S579 (2006).

[33] F. Löffler, L. Rezzolla, and M. Ansorg, Phys. Rev. D. 74, 104018 (2006).

[34] M. Shibata and K. Uryū, Phys. Rev. D. 74, 121503(R) (2006).

[35] M. Vallisneri, Phys. Rev. Lett. 84, 3519 (2000)

[36] J. W. York, Jr, Phys. Rev. Lett. 82, 1350 (1999).

[37] G. B. Cook, Living Rev. Relativity 5, 1 (2000).

[38] T. W. Baumgarte and S. L. Shapiro, Phys. Rep. 376, 41 (2003).

[39] G. B. Cook and H. P. Pfeiffer, Phys. Rev. D 70, 104016 (2004).

[40] M. Caudill, G. B. Cook, J. D. Grigsby, and H. P. Pfeiffer, Phys. Rev. D 74, 064011 (2006).

[41] E. Gourgoulhon, P. Grandclément, K. Taniguchi, J.-A. Marck, and S. Bonazzola, Phys. Rev. D 63, 064029 (2001).

[42] K. Taniguchi and E. Gourgoulhon, Phys. Rev. D 66, 104019 (2002). 
TABLE IV: Physical parameters for a binary sequence with neutron star baryon rest mass $\bar{M}_{\mathrm{B}}^{\mathrm{NS}}=0.15$. The ADM mass, and the isotropic coordinate radius of the neutron star in isolation are $\bar{M}_{\mathrm{ADM}, 0}^{\mathrm{NS}}=0.139$ and $\bar{r}_{0}=0.815(\kappa=1)$. The neutron star compaction is $M_{\mathrm{ADM}, 0}^{\mathrm{NS}} / R_{0}=0.145$ where $R_{0}$ is the areal radius of the isolated neutron star. We list the binding energy $E_{\mathrm{b}}$, total angular momentum $J$, orbital angular velocity $\Omega$, decrease of the maximum density parameter $\delta q_{\max }$, minimum of the mass-shedding indicator $\chi_{\min }$, and fractional difference $\delta M$ between the ADM mass and the Komar mass. The prefix $\dagger$ denotes the position of the turning point.

\begin{tabular}{|c|c|c|c|c|c|c|}
\hline \multicolumn{7}{|c|}{ Mass ratio: $M_{\mathrm{irr}}^{\mathrm{BH}} / M_{\mathrm{ADM}, 0}^{\mathrm{NS}}=1$} \\
\hline$d / M_{0}$ & $M_{0}$ & $E_{\mathrm{b}} / M_{0}$ & $J / M_{0}^{2}$ & $\delta q_{\max }$ & & $\delta M$ \\
\hline 15.28 & & & 1.107 & $-9.56(-5)$ & & \\
\hline & & & 1.048 & $-4.16(-4)$ & 0.934 & \\
\hline & & & 0.985 & $-1.45(-3)$ & & \\
\hline & & & 0.952 & $-2.87(-3)$ & 0.803 & \\
\hline & & & 0.933 & $-4.56(-3)$ & 0.731 & \\
\hline & & & 0.920 & $-6.43(-3)$ & 0.648 & \\
\hline & & & 0.914 & $-7.80(-3)$ & 0.575 & \\
\hline & & & 0.907 & $-1.03(-2)$ & 0.435 & \\
\hline \multicolumn{7}{|c|}{ Mass ratio: $M_{\mathrm{irr}}^{\mathrm{BH}} / M_{\mathrm{ADM}, 0}^{\mathrm{NS}}=2$} \\
\hline$d / M_{0}$ & $\Omega M_{0}$ & $E_{\mathrm{b}} / M_{0}$ & $J / M_{0}^{2}$ & $\delta q_{\max }$ & $\chi_{\min }$ & $\delta M$ \\
\hline & & & 1.089 & $-3.65(-5)$ & 0.992 & \\
\hline & & & 0.891 & $-1.19(-3)$ & 0.952 & \\
\hline & & & 0.815 & $-3.49(-3)$ & 0.870 & \\
\hline & & & 0.796 & $-5.06(-3)$ & 0.822 & \\
\hline & & & 0.777 & $-7.99(-3)$ & 0.742 & \\
\hline & & & 0.765 & $-1.12(-2)$ & 0.661 & \\
\hline & & & 0.758 & $-1.46(-2)$ & 0.566 & \\
\hline & & & 0.754 & $-1.70(-2)$ & 0.472 & \\
\hline \multicolumn{7}{|c|}{ Mass ratio: $M_{\mathrm{irr}}^{\mathrm{BH}} / M_{\mathrm{ADM}, 0}^{\mathrm{NS}}=3$} \\
\hline$d / M_{0}$ & $\Omega M_{0}$ & $E_{\mathrm{b}} / M_{0}$ & $J / M_{0}^{2}$ & $\delta q_{\max }$ & $\chi_{\min }$ & \\
\hline & & & 0.900 & $-1.01(-4)$ & 0.9 & \\
\hline & & & 0.778 & $-1.15(-3)$ & 0.981 & \\
\hline & & & 0.709 & $-2.79(-3)$ & 0.9 & \\
\hline & & & 0.661 & $-5.86(-3)$ & 0.888 & \\
\hline & & & 0.638 & $-9.61(-3)$ & 0.808 & \\
\hline & & & 0.626 & $-1.34(-2)$ & 0.735 & \\
\hline & & & 0.616 & $-2.08(-2)$ & 0.597 & 1 . \\
\hline & & -1.1 & 0.614 & $-2.31(-2)$ & 0.543 & \\
\hline \multicolumn{7}{|c|}{ Mass ratio: $M_{\mathrm{irr}}^{\mathrm{BH}} / M_{\mathrm{ADM}, 0}^{\mathrm{NS}}=5$} \\
\hline$M_{0}$ & $\Omega M_{0}$ & & $J / M_{0}^{2}$ & $\delta q_{\max }$ & $\chi_{\mathrm{m}}$ & $\delta M$ \\
\hline 18.19 & 0.0 & & 0.642 & $-3.87(-4)$ & 0.997 & 7 \\
\hline 11.64 & 0.0 & -5 . & 0.550 & $-2.12(-3)$ & 0.988 & 6. \\
\hline & & -7 & 0.481 & $-7.28(-3)$ & 0.945 & $2(-4)$ \\
\hline & 0.0617 & -8 & 0.454 & $-1.43(-2)$ & 0.856 & 2 \\
\hline & $0.0^{\circ}$ & -9. & 0.443 & $-2.15(-2)$ & 0.736 & 2.26 \\
\hline 4.37 & 0.0 & -9 & 0.442 & $-2.49(-2)$ & 0.682 & 2. \\
\hline & 0.0 & -9 & 0.441 & $-2.81(-2)$ & 0.635 & 2 \\
\hline & 0.0889 & $-9.08(-3)$ & 0.440 & $-3.23(-2)$ & 0.566 & $6(-4)$ \\
\hline \multicolumn{7}{|c|}{ Mass ratio: $M_{\mathrm{irr}}^{\mathrm{BH}} / M_{\mathrm{ADM}, 0}^{\mathrm{NS}}=10$} \\
\hline$d / M_{0}$ & $\Omega M_{0}$ & $E_{\mathrm{b}} / M_{0}$ & $J / M_{0}^{2}$ & $\delta q_{\max }$ & & $\delta M$ \\
\hline 11.91 & 0.0212 & -2.8 & 0.326 & $-2.39(-3)$ & 0.997 & $6.21(-$ \\
\hline & 0.0 & -3. & 0.291 & $-5.21(-3)$ & 0.991 & 3 \\
\hline & & & 0.276 & $-7.14(-3)$ & 0.978 & 32 \\
\hline & & & 0.269 & $-8.57(-3)$ & 0.9 & $69(-4)$ \\
\hline & & & 0.261 & $-1.19(-2)$ & 0.925 & $2.80(-4)$ \\
\hline & 0.0835 & & 0.259 & $-1.40(-2)$ & 0.904 & $2.96(-4)$ \\
\hline & & & 0.258 & $-1.67(-2)$ & 0.879 & $3.18(-4)$ \\
\hline 3.58 & 0.1028 & $-4.82(-3)$ & 0.257 & $-2.37(-2)$ & 0.823 & $3.72(-4)$ \\
\hline
\end{tabular}


[43] K. Taniguchi and E. Gourgoulhon, Phys. Rev. D 68, 124025 (2003).

[44] M. Bejger, D. Gondek-Rosińska, E. Gourgoulhon, P. Haensel, K. Taniguchi, and J. L. Zdunik, Astron. Astrophys. 431, 297 (2005).

[45] S. Bonazzola, E. Gourgoulhon, and J.-A. Marck, Phys. Rev. D 58, 104020 (1998).

[46] G. B. Cook, Phys. Rev. D 65, 084003 (2002).

[47] A. Ashtekar and B. Krishnan, Living Rev. Relativity 7, 10 (2004).

[48] E. Gourgoulhon and J. L. Jaramillo, Phys. Rep. 423, 159 (2006).

[49] LORENE web page, http://www.lorene.obspm.fr/

[50] L. Blanchet, Phys. Rev. D 65, 124009 (2002); Living Rev. Relativity 9, 4 (2006).

[51] K. Taniguchi and T. Nakamura, Phys. Rev. Lett. 84, 581 (2000); Phys. Rev. D 62, 044040 (2000).

[52] X. Zhuge, J.M. Centrella, and S. L. W. McMillan, Phys.
Rev. D 50, 6247 (1994).

[53] J. A. Faber, P. Grandclément, F. A. Rasio, and K. Taniguchi, Phys. Rev. Lett. 89, 231102 (2002).

[54] J. A. Faber, P. Grandclément, and F. A. Rasio, Phys. Rev. D 69, 124036 (2004).

[55] K. A. Dennison, T. W. Baumgarte, and H. P. Pfeiffer, Phys. Rev. D 74, 064016 (2006).

[56] In [29], we compared the values of the angular momentum with the third-order post-Newtonian result, and explained that we found "better agreement for larger separations". In fact, the opposite is correct: we actually found better agreement at smaller separations.

[57] E. Gourgoulhon, P. Grandclément, and S. Bonazzola, Phys. Rev. D 65, 044020 (2002); P. Grandclément, E. Gourgoulhon, and S. Bonazzola, Phys. Rev. D 65, 044021 (2002). 
TABLE V: Same as Table IV but for the neutron-star baryon rest mass $\bar{M}_{\mathrm{B}}^{\mathrm{NS}}=0.10$. The ADM mass, and the isotropic coordinate radius of the neutron star in isolation are $\bar{M}_{\mathrm{ADM}, 0}^{\mathrm{NS}}=0.0956$ and $\bar{r}_{0}=0.990(\kappa=1)$. The neutron star compaction is $M_{\mathrm{ADM}, 0}^{\mathrm{NS}} / R_{0}=0.0879$.

\begin{tabular}{|c|c|c|c|c|c|c|}
\hline \multicolumn{7}{|c|}{ Mass ratio: $M_{\mathrm{irr}}^{\mathrm{BH}} / M_{\mathrm{ADM}, 0}^{\mathrm{NS}}=1$} \\
\hline$d / M_{0}$ & $\Omega M_{0}$ & $E_{\mathrm{b}} / M_{0}$ & $J / M_{0}^{2}$ & $\delta q_{\max }$ & $\chi_{\min }$ & $\delta M$ \\
\hline 31.84 & 0.00533 & $-3.74(-3)$ & 1.498 & $-3.84(-5)$ & 0.976 & $4.38(-6)$ \\
\hline 25.47 & 0.00738 & $-4.59(-3)$ & 1.362 & $-2.00(-4)$ & 0.949 & $1.44(-5)$ \\
\hline 20.70 & 0.00997 & $-5.55(-3)$ & 1.250 & $-7.50(-4)$ & 0.894 & $8.77(-6)$ \\
\hline 17.51 & 0.01267 & $-6.44(-3)$ & 1.171 & $-2.21(-3)$ & 0.797 & $1.38(-6)$ \\
\hline 15.92 & 0.01453 & $-7.00(-3)$ & 1.130 & $-4.31(-3)$ & 0.683 & $1.03(-5)$ \\
\hline 15.28 & 0.01541 & $-7.24(-3)$ & 1.114 & $-5.87(-3)$ & 0.596 & $1.77(-5)$ \\
\hline 14.97 & 0.01588 & $-7.37(-3)$ & 1.106 & $-6.98(-3)$ & 0.530 & $2.41(-5)$ \\
\hline 14.81 & 0.01613 & $-7.43(-3)$ & 1.102 & $-7.68(-3)$ & 0.486 & $2.89(-5)$ \\
\hline \multicolumn{7}{|c|}{ Mass ratio: $M_{\mathrm{irr}}^{\mathrm{BH}} / M_{\mathrm{ADM}, 0}^{\mathrm{NS}}=2$} \\
\hline$d / M_{0}$ & $\Omega M_{0}$ & $E_{\mathrm{b}} / M_{0}$ & $J / M_{0}^{2}$ & $\delta q_{\max }$ & $\chi_{\min }$ & $\delta M$ \\
\hline 33.96 & 0.00483 & $-3.15(-3)$ & 1.364 & $-5.89(-5)$ & 0.990 & $1.19(-4)$ \\
\hline 21.23 & 0.00958 & $-4.85(-3)$ & 1.121 & $-4.27(-4)$ & 0.952 & $2.48(-5)$ \\
\hline 16.98 & 0.01321 & $-5.91(-3)$ & 1.027 & $-1.18(-3)$ & 0.899 & $1.50(-6)$ \\
\hline 13.80 & 0.01774 & $-7.07(-3)$ & 0.951 & $-3.57(-3)$ & 0.785 & $2.83(-6)$ \\
\hline 12.74 & 0.01986 & $-7.55(-3)$ & 0.925 & $-5.82(-3)$ & 0.699 & $1.62(-5)$ \\
\hline 12.10 & 0.02134 & $-7.88(-3)$ & 0.910 & $-8.20(-3)$ & 0.614 & $3.27(-5)$ \\
\hline 11.68 & 0.02244 & $-8.10(-3)$ & 0.899 & $-1.06(-2)$ & 0.523 & $5.11(-5)$ \\
\hline 11.46 & 0.02303 & $-8.22(-3)$ & 0.894 & $-1.23(-2)$ & 0.454 & $6.41(-5)$ \\
\hline \multicolumn{7}{|c|}{ Mass ratio: $M_{\mathrm{irr}}^{\mathrm{BH}} / M_{\mathrm{ADM}, 0}^{\mathrm{NS}}=3$} \\
\hline$d / M_{0}$ & $\Omega M_{0}$ & $E_{\mathrm{b}} / M_{0}$ & $J / M_{0}^{2}$ & $\delta q_{\max }$ & $\chi_{\min }$ & $\delta M$ \\
\hline 19.10 & 0.0111 & $-4.53(-3)$ & 0.905 & $-6.79(-4)$ & 0.961 & $8.29(-5)$ \\
\hline 15.92 & 0.0144 & $-5.31(-3)$ & 0.844 & $-1.27(-3)$ & 0.929 & $5.01(-5)$ \\
\hline 12.74 & 0.0198 & $-6.41(-3)$ & 0.779 & $-3.13(-3)$ & 0.849 & $2.51(-5)$ \\
\hline 11.94 & 0.0217 & $-6.76(-3)$ & 0.762 & $-4.22(-3)$ & 0.810 & $2.41(-5)$ \\
\hline 11.14 & 0.0239 & $-7.14(-3)$ & 0.745 & $-5.97(-3)$ & 0.753 & $2.86(-5)$ \\
\hline 10.35 & 0.0264 & $-7.57(-3)$ & 0.728 & $-9.05(-3)$ & 0.665 & $4.46(-5)$ \\
\hline 10.03 & 0.0276 & $-7.75(-3)$ & 0.721 & $-1.10(-2)$ & 0.611 & $5.70(-5)$ \\
\hline 9.55 & 0.0295 & $-8.04(-3)$ & 0.711 & $-1.52(-2)$ & 0.484 & $8.75(-5)$ \\
\hline \multicolumn{7}{|c|}{ Mass ratio: $M_{\mathrm{irr}}^{\mathrm{BH}} / M_{\mathrm{ADM}, 0}^{\mathrm{NS}}=5$} \\
\hline$d / M_{0}$ & $\Omega M_{0}$ & $E_{\mathrm{b}} / M_{0}$ & $J / M_{0}^{2}$ & $\delta q_{\max }$ & $\chi_{\min }$ & $\delta M$ \\
\hline 16.98 & 0.0131 & $-3.73(-3)$ & 0.638 & $-1.15(-3)$ & 0.975 & $1.64(-4)$ \\
\hline 12.74 & 0.0197 & $-4.79(-3)$ & 0.575 & $-2.57(-3)$ & 0.936 & $1.20(-4)$ \\
\hline 10.61 & 0.0254 & $-5.55(-3)$ & 0.542 & $-4.54(-3)$ & 0.884 & $9.89(-5)$ \\
\hline 9.55 & 0.0294 & $-6.02(-3)$ & 0.524 & $-6.55(-3)$ & 0.834 & $9.23(-5)$ \\
\hline 8.49 & 0.0345 & $-6.56(-3)$ & 0.507 & $-1.04(-2)$ & 0.748 & $9.67(-5)$ \\
\hline 7.96 & 0.0376 & $-6.85(-3)$ & 0.499 & $-1.40(-2)$ & 0.676 & $1.11(-4)$ \\
\hline 7.43 & 0.0413 & $-7.17(-3)$ & 0.491 & $-2.00(-2)$ & 0.556 & $1.41(-4)$ \\
\hline 7.33 & 0.0421 & $-7.23(-3)$ & 0.489 & $-2.17(-2)$ & 0.518 & $1.49(-4)$ \\
\hline \multicolumn{7}{|c|}{ Mass ratio: $M_{\mathrm{irr}}^{\mathrm{BH}} / M_{\mathrm{ADM}, 0}^{\mathrm{NS}}=10$} \\
\hline$d / M_{0}$ & $\Omega M_{0}$ & $E_{\mathrm{b}} / M_{0}$ & $J / M_{0}^{2}$ & $\delta q_{\max }$ & $\chi_{\min }$ & $\delta M$ \\
\hline 11.58 & 0.0224 & $-3.02(-3)$ & 0.330 & $-3.52(-3)$ & 0.976 & $1.58(-4)$ \\
\hline 8.11 & 0.0365 & $-4.00(-3)$ & 0.297 & $-8.37(-3)$ & 0.925 & $1.25(-4)$ \\
\hline 6.95 & 0.0448 & $-4.43(-3)$ & 0.286 & $-1.27(-2)$ & 0.876 & $1.16(-4)$ \\
\hline 5.79 & 0.0568 & $-4.88(-3)$ & 0.276 & $-2.22(-2)$ & 0.770 & $1.24(-4)$ \\
\hline 5.50 & 0.0607 & $-4.98(-3)$ & 0.273 & $-2.67(-2)$ & 0.723 & $1.34(-4)$ \\
\hline 5.21 & 0.0650 & $-5.07(-3)$ & 0.271 & $-3.30(-2)$ & 0.657 & $1.45(-4)$ \\
\hline 4.98 & 0.0688 & $-5.13(-3)$ & 0.270 & $-3.99(-2)$ & 0.579 & $1.54(-4)$ \\
\hline 4.93 & 0.0698 & $-5.14(-3)$ & 0.269 & $-4.16(-2)$ & 0.553 & $1.55(-4)$ \\
\hline
\end{tabular}

